\title{
Retrieval of water vapor using ground-based observations from a prototype ATOMMS active centimeter- and millimeter-wavelength occultation instrument
}

\author{
Dale M. Ward ${ }^{1}$, E. Robert Kursinski ${ }^{2}$, Angel C. Otarola ${ }^{1,3}$, Michael Stovern ${ }^{4}$, Josh McGhee ${ }^{2}$, Abe Young ${ }^{5}$, \\ Jared Hainsworth $^{6}$, Jeff Hagen ${ }^{7}$, William Sisk ${ }^{8}$, and Heather Reed ${ }^{9}$ \\ ${ }^{1}$ Department of Atmospheric Sciences, University of Arizona, Tucson, AZ 85721, USA \\ ${ }^{2}$ PlanetIQ, Golden, CO 80401, USA \\ ${ }^{3}$ TMT International Observatory, LLC., Pasadena, CA 91124, USA \\ ${ }^{4}$ Environmental Protection Agency, Denver, CO 80202, USA \\ ${ }^{5}$ Department of Physics, University of Arizona, Tucson, AZ 85721, USA \\ ${ }^{6}$ Hill Air Force Base, A-10 Mechanical Systems, Ogden, UT 84056, USA \\ ${ }^{7}$ Lithe Technology, Tucson, AZ 85721, USA \\ ${ }^{8}$ Department of Astronomy, University of Arizona, Tucson, AZ 85721, USA \\ ${ }^{9}$ LASP, University of Colorado, Boulder, CO 80303, USA
}

Correspondence: Dale M. Ward (dward@email.arizona.edu)

Received: 19 May 2017 - Discussion started: 26 July 2017

Revised: 6 October 2018 - Accepted: 10 October 2018 - Published: 27 March 2019

\begin{abstract}
A fundamental goal of satellite weather and climate observations is profiling the atmosphere with in situlike precision and resolution with absolute accuracy and unbiased, all-weather, global coverage. While GPS radio occultation (RO) has perhaps come closest in terms of profiling the gas state from orbit, it does not provide sufficient information to simultaneously profile water vapor and temperature. We have been developing the Active Temperature, Ozone and Moisture Microwave Spectrometer (ATOMMS) RO system that probes the 22 and $183 \mathrm{GHz}$ water vapor absorption lines to simultaneously profile temperature and water vapor from the lower troposphere to the mesopause. Using an ATOMMS instrument prototype between two mountaintops, we have demonstrated its ability to penetrate through water vapor, clouds and rain up to optical depths of 17 (7 orders of magnitude reduction in signal power) and still isolate the vapor absorption line spectrum to retrieve water vapor with a random uncertainty of less than $1 \%$. This demonstration represents a key step toward an orbiting ATOMMS system for weather, climate and constraining processes. ATOMMS water vapor retrievals from orbit will not be biased by climatological or first-guess constraints and will be capable of capturing nearly the full range of variability through the at-
\end{abstract}

mosphere and around the globe, in both clear and cloudy conditions, and will therefore greatly improve our understanding and analysis of water vapor. This information can be used to improve weather and climate models through constraints on and refinement of processes affecting and affected by water vapor.

\section{Introduction (motivation)}

Water vapor is an important constituent in the Earth's atmosphere and its distribution in space and time must be known to understand and predict weather and climate. Water vapor is fundamental to the radiative balance of the Earth, both as the most important greenhouse gas and indirectly through clouds. Through its latent heat, water vapor is crucial to the formation and evolution of severe weather, transport of energy both upward and poleward in the troposphere and transfer of energy between the surface and atmosphere. Furthermore, water vapor dominates tropospheric radiative cooling, which drives convection (Sherwood et al., 2010). Uncertainty in modeled cloud feedback results in the spread of a factor 
of 3 in predictions of the surface temperature response to a doubling of atmospheric $\mathrm{CO}_{2}$ concentrations, and the cloud feedback depends critically on the strength of the water vapor feedback (Held and Soden, 2000). Predicted amplification of extreme precipitation with warmer temperatures is tied directly to predicted increases in extreme water vapor concentrations and future extreme precipitation may be underestimated (e.g., Allan and Soden, 2008).

Water vapor is challenging to measure because of the wide range of concentrations and scales across which it varies. Water vapor observations must be unbiased and capture the full range of variability in clear and cloudy conditions across the globe in order to improve the understanding and analysis of water vapor, This information is used to initialize weather prediction systems, to monitor trends and variations and to improve weather and climate models through constraints on and refinement of processes affecting and affected by water vapor (e.g., Bony et al., 2015).

Satellite observations are required to gain a global perspective of weather prediction and climate monitoring and for constraining the critical processes at work in different regions across the globe. Unfortunately, present satellite observations provide limited constraints on the water vapor field, particularly when clouds are present, which in turn limits the capability of weather forecasts and our detailed knowledge of water vapor across the globe. For example, GOES observations provide high time and horizontal resolution but very limited vertical information. While hyperspectral IR on polar orbiting satellites provide more information, their temporal sampling is limited and their water vapor estimates are quite noisy with fractional, root mean square (rms) differences ranging from $25 \%$ in the lower troposphere to $70 \%$ around $400 \mathrm{hPa}$ and a tendency toward dry biases up to $30 \%$, depending on cloud type (Wong et al., 2015). While downwardlooking microwave radiance measurements are particularly useful for determining the column water over the ocean (e.g., Wang et al., 2016), they provide significantly less vertical information than IR and are inherently ambiguous over land, snow and ice due to surface emissivity variations. The point is that present state-of-the-art, radiance-based satellite water vapor remote-sensing systems have serious limitations in terms of performance and sampling biases associated with clouds and surface conditions, accuracy, vertical resolution and the ambiguity inherent in the conversion of radiances to the atmospheric state (Rodgers, 2000).

Because of these satellite limitations, balloon-borne sondes and dropsondes continue to be the measurement of choice for field campaigns focused on answering key questions about the atmosphere. In fact, the globe would be covered with sondes if the cost to do so were not so completely prohibitive. Operational global weather observing systems therefore rely primarily on more affordable but vertically coarse satellite radiance measurements and the inherent ambiguities in the information they provide. Unfortunately, this limits how much understanding we can gain from these ob- servations about important atmospheric processes like those associated with clouds, convection and surface exchange.

In this context, GPS radio occultation (RO) has provided a welcome advance in satellite remote sensing through its ability to profile the atmosphere with $\sim 200 \mathrm{~m}$ vertical resolution, approaching that of sondes, in all-weather conditions, with very small random and absolute uncertainties. As such, GPS RO has become an important data source for numerical weather prediction (NWP), despite its relatively sparse coverage to date (e.g., Cardinali and Healy, 2014). Its high impact comes from its unique combination of $\sim 200 \mathrm{~m}$ vertical resolution, all-weather sampling and very low random and absolute uncertainties via its direct connection to atomic frequency standards and relatively simple and direct retrieval methods. GPS RO profiles atmospheric refractivity. Two limitations of GPS RO are (1) its inability to separate the dry-air and water vapor contributions to refractivity and (2) its insensitivity to water vapor in the colder regions of the troposphere and above (e.g., Kursinski et al., 1997; Kursinski and Gebhardt, 2014). The insensitivity occurs when there is so little water vapor that the majority of the refractivity is dominated by the dry-air component.

In recognition of the strengths and weaknesses of GPS RO and radiance measurements as well as the need for better information about water vapor, in 1997 research groups at the University of Arizona and the NASA Jet Propulsion Laboratory (Herman et al., 1997; Hajj et al., 1997) identified and began developing an RO system that is now called the Active Temperature, Ozone and Moisture Microwave Spectrometer (ATOMMS), which is designed to overcome these GPS limitations by transmitting and receiving signals between satellites in low Earth orbit (LEO) near the 22 and $183 \mathrm{GHz}$ water vapor absorption lines as well as nearby ozone absorption lines. Profiling both the speed of light like GPS RO as well as the absorption of light, which GPS RO does not measure, enables ATOMMS to profile temperature, pressure and water vapor simultaneously from near the surface to the mesopause with little random or systematic uncertainty (Kursinski et al., 2002). It will also profile ozone from the upper troposphere into the mesosphere, scintillations produced by turbulence, slant path cloud liquid water and will detect larger cloud ice particles with approximately $100 \mathrm{~m}$ vertical resolution and corresponding $70 \mathrm{~km}$ horizontal resolution (Eq. 13, Kursinski et al., 1997). Kursinski et al. (2002) found that such a system could provide water vapor retrievals with a random uncertainty of 1\%-3\% from near the surface to well into the mesosphere. Kursinski et al. (2009) estimated that the degradation in clouds would be less than a factor of 2 . A summary of LEO-to-LEO occultation measurement concept studies and demonstrations to date at microwave and IR wavelengths is given in Liu et al. (2017).

Regarding the sampling densities that can be achieved with ATOMMS, Kursinski et al. (2016b) noted that a constellation of 60 very small satellites, carrying both ATOMMS and GNSS RO sensors, would produce approximately 26000 
ATOMMS and 170000 GNSS occultations profiles each day, for a fraction of the cost of a single, operational, polarorbiting weather satellite. These numbers of profiles are approximately 10 and 100 times present GPS RO and radiosonde sampling densities. Such an orbiting ATOMMS constellation providing dense, very high vertical resolution, precision and accuracy water and temperature profiling via radio occultation will complement existing observations of clouds, precipitation and energy fluxes and tie the entire weather and climate system together. This combination will also dramatically improve the realism and utility of global analyses for climate as well as forecasting (increasingly extreme) weather (Kursinski et al., 2016a).

With regard to constraining processes, we briefly discuss three important and representative application areas: moist convection, weather fronts and polar weather and climate.

\subsection{Moist convection}

Moist convection is ubiquitous across the globe but inadequately understood which leads to inaccurate representation in models. Environmental variables critical for understanding and predicting moist convection and associated severe weather include temperature, water vapor, stability, conditional instability in particular, the level of free convection, convective available potential energy (CAPE), convective inhibition (CIN), winds and divergence. Unfortunately, coarse vertical resolution and ambiguities inherent in converting radiance spectra to the atmospheric state limit the ability of satellite radiances to provide detailed constraints on convection-related processes. GPS RO provides muchneeded vertical information across the globe and is particularly useful for determining temperatures and stability in the upper troposphere where conditions are very dry. However, the ambiguity of the wet and dry gas contributions to refractivity under the warmer, moister conditions deeper in the troposphere limit the utility of GPS RO refractivity profiles there.

In contrast, ATOMMS will be the first orbiting remotesensing system to simultaneously profile temperature and water vapor with very high $\sim 100 \mathrm{~m}$ vertical resolution and very small uncertainties needed to tightly constrain these environmental quantities relevant to convection, in clear and cloudy conditions, through the troposphere and across the entire globe. While ATOMMS profiles will not resolve detailed horizontal structure at scales much below $70 \mathrm{~km}$, they are sensitive to these scales via the phase and amplitude scintillations that small-scale turbulence produces on the ATOMMS signals (Kursinski et al., 2016b). Furthermore, $100 \mathrm{~km}$, which is approximately the horizontal resolution of ATOMMS, is the scale most important for forecasting severe convection in the form of thunderstorms (Durran and Weyn, 2016).

\subsection{Weather fronts}

Weather fronts are another fundamental class of severe weather poorly constrained by satellite radiance measurements. Unlike radiances, RO measurements can profile fronts from orbit because RO profiles readily penetrate through clouds and the vertical and horizontal resolutions of RO are well matched to the vertical and horizontal scales of weather fronts. While GPS RO can profile fronts in the upper troposphere (e.g., Kuo et al., 1998), the lack of refractivity contrast between the warm-wet and the cold-dry sides of fronts deeper in the troposphere limits GPS RO profiling of fronts there (Hardy et al., 1994). ATOMMS high-precision temperature, pressure and water vapor profiles in clear and cloudy conditions will readily distinguish between the warm and cold sides of fronts down through the lower troposphere and precisely determine the location of any frontal surface that crosses an ATOMMS profile (Kursinski et al., 2002).

This unprecedented capability to measure fronts globally will also enable detailed characterization of the dynamics and moisture fluxes of atmospheric rivers out over remote ocean regions to better predict and prepare for the torrential rainfall and flooding they produce following landfall. These observations will also guide refinements in model representations of atmospheric rivers to increase and extend the accuracy of weather forecasts and the climatologically important midlatitude water vapor transport in reanalyses and climate models (e.g., Guan and Waliser, 2016).

\subsection{Profiling in polar regions}

Profiling in polar regions, particularly the near-surface environment, is critical to understanding the causes of ongoing and future climatic changes there. Reducing uncertainty due to our limited knowledge about the critical processes at work there requires quantitative, process-resolving observations that span the entire range of environmental conditions and behavior across these remote regions. Present understanding comes largely from operational sondes and a small number of field campaigns (e.g., Esau and Sorokina, 2010). While satellites radiance measurements already provide dense sampling of these remote, high-latitude regions, they have yielded relatively little insight due to intrinsic ambiguities associated with poor vertical resolution, frequent clouds, near-surface inversions and variations in surface emissivity. As a result, many "global" satellite products do not extend to the poles (e.g., Chen et al., 2008). While GPS RO has much-needed very high vertical resolution, cloud penetration and insensitivity to surface conditions, its impact is also limited, because of the unknown contributions of water vapor and the bulk dry gas to the measured refractivity profiles.

In this context, precise and very high vertical resolution ATOMMS profiling of temperature, stability, water vapor, pressure gradients, clouds and turbulence, down to the surface, over all types of surfaces, in clear and cloudy condi- 
tions, across the diurnal and seasonal cycles, will bring unprecedented information about the high latitudes and, in particular, the lowermost troposphere, to constrain and reduce presently large uncertainties in surface fluxes and the surface energy budget there.

ATOMMS will simultaneously probe through clouds to determine the gas state as well as the cloud properties themselves, including their phases (liquid, ice and mixed), which are critical in the surface energy budget (e.g., Klingebiel et al., 2015) and fundamental to calculating upward and downward short- and long-wave radiative fluxes through the atmosphere. ATOMMS will profile the frequent polar boundary layer clouds that are too close to the surface to be characterized by CloudSat (Kay and Gettleman, 2009).

ATOMMS will constrain winds via horizontal pressure gradients to further constrain wind shear and moisture fluxes. This wind and cloud information, together with simultaneous profiling by ATOMMS of stability and turbulent scintillations, will provide a new set of observational constraints over the entire high-latitude region to expose flaws and guide improvements to presently inaccurate and poorly constrained model parameterizations of sensible and latent heat fluxes. The ability to estimate turbulence and radiative cooling at cloud top are also critical to determining cloud lifetimes and the radiative budget because turbulent entrainment rates influence droplet size and therefore albedo (Esau and Sorokina, 2010). ATOMMS global perspective would provide critical information for understanding why the two poles are evolving so differently.

The preceding examples reveal inadequacies in our present observing system that limit our understanding and in the substantial increase that ATOMMS promises in our observationally based knowledge and understanding. The performance of ATOMMS profiles approaches that of sondes and, when implemented as a constellation such as in Kursinski et al. (2016b), would provide far denser coverage across the globe. For example, the vast Amazon rainforest which is presently profiled twice a day by only 8 sondes (Itterly et al., 2016), would be sampled by approximately 300 ATOMMS profiles and 1800 GNSS RO profiles each day via the ATOMMS satellite constellation noted above. Thus, an ATOMMS constellation would create a continuous, dense, global data set, with performance approaching that of sondes, which researchers could divide up as they like into smaller domains (creating essentially their own regional (field) campaigns) to better understand and model key processes and reduce weather and climate prediction uncertainty across the globe.

Our work here is focused on a mountaintop demonstration of the ability of ATOMMS to measure water vapor through rain and clouds. Using ground-based ATOMMS prototype instrumentation, we demonstrate the ability of ATOMMS to retrieve changes in the path-averaged water vapor between the instruments operating between two mountaintops in Southern Arizona to within 1\%, during weather condi- tions that ranged from clear to cloudy to thunderstorms with heavy rain. The ATOMMS mountaintop retrievals worked up to optical depths of 17. The discrepancies smaller than $1 \%$ between the measured ATOMMS spectra and the forward modeled water vapor spectra (described in Sect. 4), in clear, cloudy and rainy conditions are unprecedented and more than 1 order of magnitude smaller than the $25 \%$ to $70 \%$ uncertainties in AIRS retrievals reported in Wong et al. (2015). At still higher cloud and rain opacities, such as the conditions encountered during our ATOMMS mountaintop experiment, IR and microwave emission-based water vapor retrievals simply do not work. ATOMMS performance in cloud and rain is achieved via a differential transmission approach using a calibration signal, in contrast to passive IR and microwave sensors systems that work via emission. In addition, the vertical resolution attainable via active occultation observing systems is at least an order of magnitude better than that of passive sensors.

The structure of the paper is as follows. Section 2 summarizes the ATOMMS concept for satellites operating at low Earth orbit (LEO) and Sect. 3 describes this mountaintop experiment. In Sect. 4, we discuss the water vapor retrievals from the measured mountaintop data. Sources of uncertainty are covered in Sect. 5, while Sect. 6 examines validation of the water vapor retrievals with available in situ measurements. Finally, in Sect. 7, the encouraging results from the ATOMMS ground-based system lead us to a discussion of the unique capabilities of a future ATOMMS satellite occultation system for improving numerical weather forecasts, monitoring climate changes and improving our understanding and model representation of processes related to water vapor.

\section{ATOMMS concept}

ATOMMS is a natural extension of the GPS RO concept. It extends the capabilities and overcomes several limitations of GPS RO by simultaneously measuring atmospheric bending and absorption at several essentially monochromatic signal frequencies in two frequency bands centered on the 22 and $183 \mathrm{GHz}$ water absorption lines, referred to as low-band and high-band, respectively. The high-band includes several ozone absorption lines used to profile ozone. During ATOMMS satellite-to-satellite occultations, signals transmitted from one satellite are received by the other, which yields measurements of the signal phase and amplitude during the occultation. From these, occultation profiles of bending angle and absorption are derived and then used to derive radial profiles of refractivity and the extinction coefficient using Abel transforms (Kursinski et al., 2002). These are then combined with knowledge of spectroscopy, together with the equations of refractivity and hydrostatic equilibrium, to derive profiles of air temperature, pressure, water vapor, ozone and some properties of condensed water. 
ATOMMS functions as a precise, active spectrometer over the propagation path between the transmitter and receiver. Retrievals of water vapor from radiance measurements are inherently ambiguous because both the unknown signal source emission and attenuation along the path are unknown and must be solved for, creating an ill-posed problem (e.g., Rodgers, 2000). In comparison to radiance retrievals, ATOMMS has the advantage that the transmitted signal strength is well known and the observed quantity is simply the attenuation along the path, which makes the retrievals much more direct and less ambiguous. The active approach also enables retrievals with small random and systematic uncertainty under conditions of large path optical depths, which is not possible for passive retrievals.

Because ATOMMS uses phase coherent signals to measure Doppler shift and bending angle like GPS RO, we write the signal attenuation in terms of amplitude rather than intensity as follows:

$A(f)=A_{0}(f) e^{-\tau / 2}$,

where $A$ is the measured signal amplitude after the absorption, $A_{0}$ is the amplitude of the signal that would be measured in the absence of atmospheric attenuation and $\tau$ is the optical depth at the signal frequency, $f$. The factor of 0.5 multiplying the optical depth comes about because intensity is proportional to amplitude squared. The total optical depth is due to the gas-phase optical depth plus the attenuation due to hydrometeors. The gas-phase optical depth includes water vapor and dry-air absorption, which depend on temperature and pressure. The hydrometeor attenuation also depends on temperature (Kursinski et al., 2009).

\section{Differential absorption}

A key to ATOMMS performance is its double differential absorption approach (Kursinski et al., 2002). First, the amplitude observable is the change in signal amplitude over an occultation relative to the amplitude measured at time, $t_{0}$, when the signal path between the two spacecraft is entirely above the atmosphere. Second, the amplitudes of two (or more) signals are measured simultaneously during each occultation. The frequency, $f$, of one signal is placed on the absorption line of interest, while the frequency of the second signal, $f_{\mathrm{CAL}}$, is farther from the line center, so that signal can function as an amplitude calibration signal.

The quantity used in the ATOMMS retrievals is the ratio of two amplitude ratios,

$R\left(f, f_{\mathrm{CAL}}, t, t_{0}\right)=\frac{A(f, t)}{A\left(f_{\mathrm{CAL}}, t\right)} / \frac{A\left(f, t_{0}\right)}{A\left(f_{\mathrm{CAL}}, t_{0}\right)}$.

The amplitude ratio in the denominator represents the ratio of the amplitude of the tuned signal to the amplitude of the calibration signal at reference time, $t_{0}$, when the signal is nominally above the atmosphere. The amplitude ratio in the nu- merator represents the ratio of the amplitude of the tuned signal to the amplitude of the calibration signal at measurement time, $t$, during the occultation. Taking the natural logarithm of $R$ and multiplying by two yields the change in the difference between the optical depths at frequencies $f$ and $f_{\mathrm{CAL}}$, from the reference time, $t_{0}$, to time, $t$.

$2 \log (R)=\tau(f, t)-\tau\left(f_{\mathrm{CAL}}, t\right)-\left[\tau\left(f, t_{0}\right)-\tau\left(f_{\mathrm{CAL}}, t_{0}\right)\right]$

If the signal path is entirely above the atmosphere at reference time, $t_{0}$, as will be the case in a LEO-LEO occultation geometry, then the optical depths at time $t_{0}$ are zero and Eq. (3) simplifies to

$2 \log (R)=\tau(f, t)-\tau\left(f_{\mathrm{CAL}}, t\right)$.

The frequency separation between $f$ and $f_{\mathrm{CAL}}$ is chosen such that $R$ retains most of the absorption signature while canceling unwanted common sources of error such as gain variations due to pointing errors, scintillations due to atmospheric turbulence and attenuation due to scattering by hydrometeors. This ratio of ratios approach enables precise measurements of water vapor in the presence of clouds and rain with very small random and systematic uncertainty as we demonstrate below.

\section{Overview of the ATOMMS mountaintop experiment}

We designed and built a ground-based, prototype ATOMMS instrument and then used it to demonstrate some key aspects of ATOMMS capabilities and performance in several fixed geometries in southern Arizona with path lengths ranging from $800 \mathrm{~m}$ to $84 \mathrm{~km}$. The prototype ATOMMS high-band system transmits and receives two simultaneous continuouswave $(\mathrm{CW})$ signals tunable from 181 to $206 \mathrm{GHz}$. The prototype low-band system consists of eight $\mathrm{CW}$ transmitters and receivers at fixed frequencies from 18.5 to $25.5 \mathrm{GHz}$ spaced approximately $1 \mathrm{GHz}$ apart, centered approximately on the $22 \mathrm{GHz}$ water vapor absorption line. Below we summarize the content of previous published work based on field experiments with the ATOMMS ground-based prototype.

In terms of ATOMMS water vapor retrievals, Kursinski et al. (2012) demonstrated agreement at the $2 \%$ level between water vapor measurements derived along an $820 \mathrm{~m}$ path using the ATOMMS high-band instrument and a nearby, capacitive-type hygrometer. High-band mountaintop measurements yielded the first detection by ATOMMS of $\mathrm{H}_{2}^{18} \mathrm{O}$ via its $203 \mathrm{GHz}$ absorption line (Kursinski et al., 2016b). Such measurements in the upper troposphere will determine isotopic ratios to constrain the hydrological cycle (Kursinski et al., 2004).

Accurate knowledge of spectroscopy is key to interpreting the ATOMMS measurements. ATOMMS itself is perhaps the best $183 \mathrm{GHz}$ spectrometer ever implemented. Its measurements of the line shape near the $183 \mathrm{GHz}$ line center 
match that of the HITRAN model to within $0.3 \%$ (Kursinski et al., 2012), which agrees 8 times better than the best prior estimates of Payne et al. (2008). These same measurements revealed that the line shape of the popular Liebe et al. (1993) model is incorrect (Kursinski et al., 2012). Farther from the line center, 5 to $25 \mathrm{GHz}$ above the line center, ATOMMS measurements revealed significant discrepancies with the HITRAN line shape (Kursinski et al., 2016b). These discrepancies may help explain inconsistencies between 183 $\mathrm{GHz}$ derived water vapor estimates discussed in Brogniez et al. (2016) that may be associated with atmospheric turbulence (Calbet et al., 2018).

In terms of sensing hydrometeors, Kursinski et al. (2012) derived cloud liquid water content (LWC) by combining ATOMMS high-band measurements with precipitation radar measurements along the ATOMMS signal path. Kursinski et al. (2016b) further demonstrated the ability to derive both cloud LWC and rainfall rates by combining the ATOMMS low-band and high-band measurements. ATOMMS also acts as a scintillometer to sense atmospheric turbulence. Kursinski et al. (2016b) derived the strength of atmospheric turbulence from scintillations of the ATOMMS signal amplitudes and further demonstrated how these turbulent amplitude variations can be reduced via amplitude ratioing, as needed to derive accurate water vapor estimates in turbulent conditions.

On 18 August 2011, we collected approximately $4 \mathrm{~h}$ of data with the instruments located on Mt. Lemmon Ridge (2752 $\mathrm{m}$ altitude) and Mt. Bigelow (2515 m altitude), separated by approximately $5.4 \mathrm{~km}$. The observing geometry is shown in Fig. 1. The Mt. Lemmon instrument contained the $183 \mathrm{GHz}$ transmitter and $22 \mathrm{GHz}$ receiver and the Mt. Bigelow instrument contained the $22 \mathrm{GHz}$ transmitter and $183 \mathrm{GHz}$ receiver. The water vapor pressure derived from these ATOMMS measurements represents an average over the $5.4 \mathrm{~km}$ path, which runs above a valley between the mountaintops on which the instruments sit.

\subsection{Differences between mountaintop and LEO measurements}

The mountaintop-to-mountaintop geometry differs from the satellite-to-satellite geometry in several important aspects. In the satellite-to-satellite occultation geometry, the ATOMMS differential absorption measurements yield absolute water vapor concentrations because the reference signal strength is measured above the atmosphere where there is no absorption. Since we cannot evacuate the path between the two mountaintops, mountaintop-to-mountaintop observations are limited to measuring changes in water vapor relative to a selected reference period as defined in Eq. (3). In the satellite geometry, a profile of water vapor is retrieved as a function of altitude via an Abel transform (Kursinski et al., 2002). In the mountaintop experiment, the signal path is fixed and the retrieved quantity is the change in the average water vapor along the fixed path as a function of time.
In the satellite-to-satellite occultation geometry, the majority of the signal attenuation occurs along the lowest altitude portion of the signal path centered at the ray tangent point, which is 100 to $500 \mathrm{~km}$ in length. The attenuation contributed at higher altitudes along the ray path is comparatively much smaller than the contribution near the ray path tangent altitude due to both the limb-sounding geometry and the exponential decay in water vapor concentrations with altitude. We note that the Abel transform isolates the contribution from the lowest altitude portion of the signal path. For a vertical resolution of $100 \mathrm{~m}$, the horizontal length of the path through the lowest layer is approximately $70 \mathrm{~km}$ (Eq. 13, Kursinski et al., 2002). Because the large water vapor concentrations in the lower and middle troposphere produce impenetrably high opacities near the $183 \mathrm{GHz}$ line when integrated over such long signal paths, this portion of the troposphere must be profiled using the weak $22 \mathrm{GHz}$ absorption line and the ATOMMS low-band system from space. This is also the altitude region where liquid water clouds are most common. To achieve our goal of an all-weather observing system, the observations must provide enough information for the inversion routine to be able to separate the signal attenuation due to liquid water absorption from that due to water vapor absorption. Kursinski et al. (2009) showed that the spectral shape of the cloud liquid water absorption at the low-band frequencies depends primarily on the cloud liquid water path and cloud temperature. Simultaneously measuring the amplitudes of four low-band signals, with at least one of the signal frequencies on the high side of the $22 \mathrm{GHz}$ line, in addition to refractivity plus application of a hydrostatic constraint, enables water vapor, cloud liquid water path and effective cloud temperature to be estimated simultaneously. Thus, with absorption information from at least four low-band frequencies, we can isolate liquid water clouds from water vapor and unwanted variations due to instrumental noise and turbulence. Simulations in Kursinski et al. (2009) showed that the uncertainty in cloudy conditions should increase by no more than a factor of 2 relative to clear-sky conditions. We also note that Kursinski et al. (2009) recommended using at least five signal frequencies in order to expose spectral modeling errors and provide the quantitative information needed to refine the modeling of both the water vapor and liquid water spectra.

In this mountaintop demonstration, the atmospheric path from transmitter to receiver took place over a narrow altitude range from 2752 to $2515 \mathrm{~m}$ above sea level and was only $5.4 \mathrm{~km}$ in length. Over this short path, the water vapor attenuation due to absorption by the weak $22 \mathrm{GHz}$ line was too small to measure accurately. Therefore, in this experiment, we used the ATOMMS high-band signals to probe near the stronger $183 \mathrm{GHz}$ water line to retrieve changes in water vapor along the path. Below we show that the liquid attenuation has a relatively flat spectral response across the high-band frequencies utilized for the mountaintop retrieval of water vapor and essentially ratios out. In the satellite case, at altitudes where liquid clouds commonly occur, the combined attenua- 


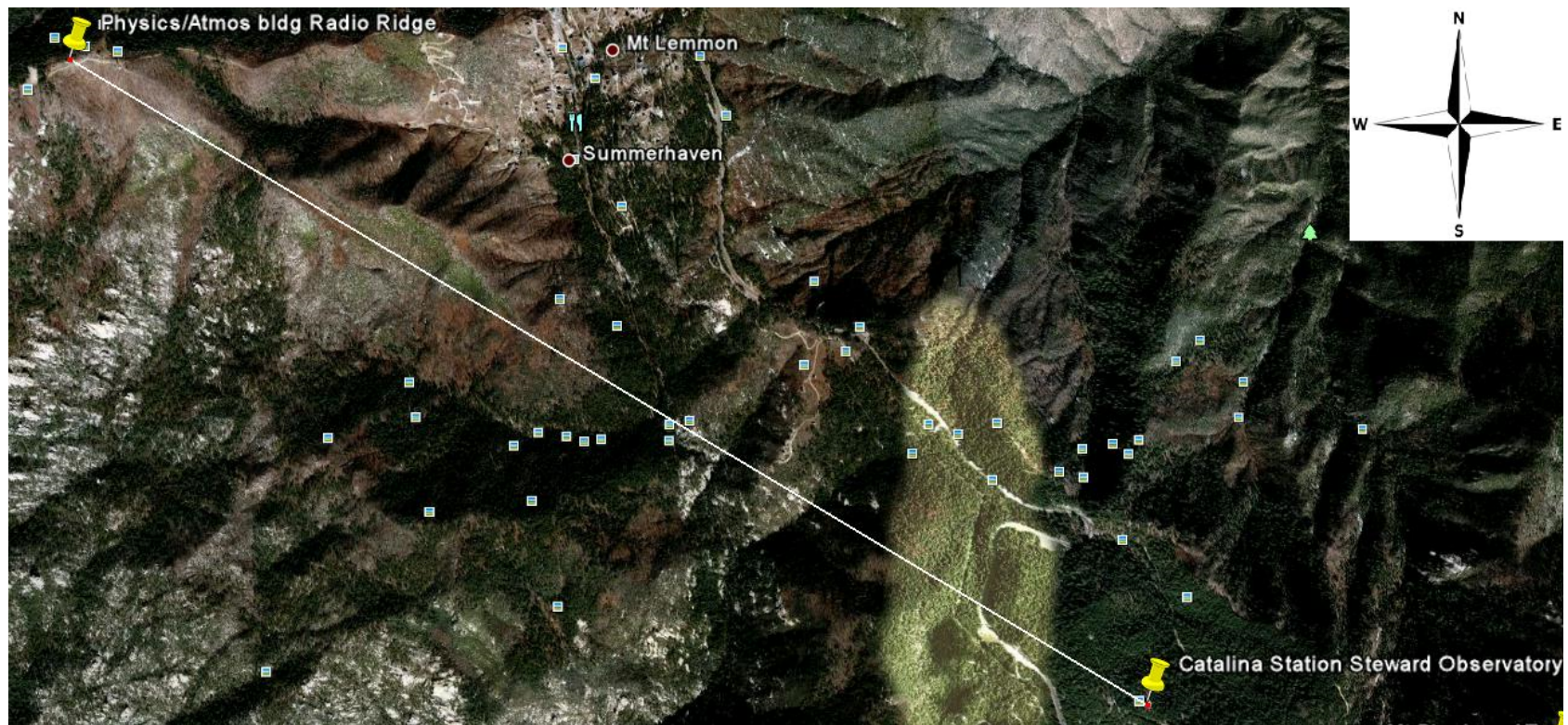

Figure 1. Geometry for the ATOMMS ground-based prototype instrument tests. The high-band transmitter was located on Radio Ridge near Mt. Lemmon at an altitude of $2752 \mathrm{~m}$, and the high-band receiver was located $5.4 \mathrm{~km}$ away at the Steward Observatory Catalina Station near Mt. Bigelow at an altitude of $2515 \mathrm{~m}$. The signal propagation path lies along a northwest-to-southeast line.

tion from liquid water and water vapor will make the atmosphere too opaque to probe with the high-band frequencies and ATOMMS will therefore profile these conditions with the low-band signals near the $22 \mathrm{GHz}$ line as noted above.

Another difference is that in the LEO-LEO geometry, profiles of atmospheric refractivity and temperature are derived from a Doppler shift proportional to atmospheric bending (e.g., Kursinski et al., 1997). In a fixed geometry, there is no equivalent Doppler shift and we therefore had to determine the air temperature via another method which is described in Sect. 4 .

A final point relates to instrument stability. The duration of a typical LEO-LEO occultation is approximately $100 \mathrm{~s}$, which allows little time for instrument drift, while mountaintop measurements can continue for hours or days. Therefore, to maintain instrument stability over the $4 \mathrm{~h}$ mountaintop observation period, we used water chillers to minimize temperature variations in critical portions of the transmitters and receivers.

In spite of the differences noted above, this ground-based experiment clearly demonstrates the ability of an ATOMMStype system to probe through and accurately retrieve changes in water vapor under conditions of large total optical depths with liquid water present along the path.

\subsection{Observed optical depths}

The measured changes in optical depth at $198.5 \mathrm{GHz}$ (blue line, raw) and $24.4 \mathrm{GHz}$ (red line, raw) are shown in Fig. 2. $198.5 \mathrm{GHz}$ was the frequency of the high-band calibration signal during this experiment. Also shown are the de- rived changes in liquid optical depth at $198.5 \mathrm{GHz}$ (black line), which was computed by subtracting the optical depth changes due to variations in the retrieved vapor pressure and temperature from the total observed optical depth change. The change in optical depth relative to reference period 1 will always be positive for liquid (rain and clouds), because there was no rain or clouds during the reference period. However, the change in optical depth due to changes in vapor pressure and temperature can be negative, which means that the overall change in optical depth relative to the reference period can be less than the optical depth change due to liquid alone.

The instruments were housed in tents to protect them from weather conditions that spanned from clear to cloudy to thunderstorms with heavy rain, as indicated by the annotations in Fig. 2. This wide range of conditions and associated optical depths provided an excellent field test to evaluate and demonstrate several key ATOMMS capabilities. In situ measurements of temperature, pressure and water vapor were made at each tent. Web cameras in each tent pointed at the opposite ATOMMS instrument site, providing periodic images of weather conditions and visible opacity.

Figure 2 indicates that when the ATOMMS observations began, a light rain was falling. The rain ended prior to the first reference period. A brief rain shower was observed from about 14:43 to 15:02. The sharp peak in the $198.5 \mathrm{GHz}$ liquid optical depth just before 15:00 and absence of a peak in the $24.4 \mathrm{GHz}$ liquid optical depth likely indicates an increase in the number of smaller raindrops. This was followed by a brief clear period before the next rain shower began at 15:10. This rain was initially light, but became a heavy thunderstorm at 


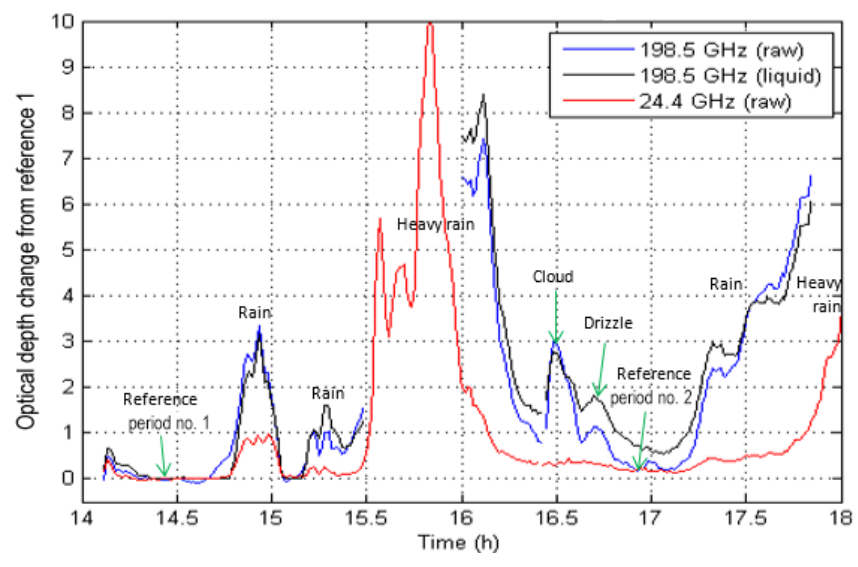

Figure 2. Blue and red lines show observed changes in optical depth at 198.5 and $24.4 \mathrm{GHz}$ relative to reference period 1 . The black line shows changes in optical depth at $198.5 \mathrm{GHz}$ due to changes in liquid water after removing the contribution from changes in vapor pressure and temperature.

15:30. From $15: 30$ to $16: 00$, the $198.5 \mathrm{GHz}$ tone was too attenuated to be observed at the receiver. During the heavy rain, the $24.4 \mathrm{GHz}$ liquid optical depth reached a peak value of 10 . The $198.5 \mathrm{GHz}$ signal was detected again at 16:00 as the rain lightened. By 16:30, the rain was considerably lighter. The radar data from the Tucson WSR-88D radar (Crum and Alberty, 1993) and field observations indicated that rain was still falling over portions of the path between the two instruments. Note that the liquid optical depths did not return to zero before the next heavier rain shower began, around 17:15.

Between 16:28 and 16:31, a cloud advected through the observation path. Field notes and images taken every $30 \mathrm{~s}$ show a cloud moving into and through the field of view. Initially the cloud extended only part way across the observation path. It then apparently spanned the entire path for a brief period of less than 2 min before gradually clearing out of the observation path. The presence of smaller cloud droplets caused the $198.5 \mathrm{GHz}$ liquid optical depth to increase around $16: 30$, while little if any change was apparent in the $24.4 \mathrm{GHz}$ liquid optical depth. The fact that the $24.4 \mathrm{GHz}$ optical depth did not drop to 0 indicates some light rain was present as well. The decrease in $198.5 \mathrm{GHz}$ liquid optical depth after the peak at 16:30 likely indicates that cloud droplets or drizzle obscured only part of the observation path.

\subsection{Signal tuning and detection}

The high-band portion of the ATOMMS ground-based prototype instrument simultaneously transmits and receives two continuous-wave signals that are tunable from 181 to $206 \mathrm{GHz}$. For this mountaintop experiment, the frequency of the signal generated by one transmitter was swept through a tuning sequence that spanned the instrument's tunable frequency range. This signal was received by a narrowband het- erodyne receiver with a second local oscillator that was simultaneously swept through its matching tuning sequence. The frequency of the other signal was fixed at $198.5 \mathrm{GHz}$ in order to function as the amplitude calibration signal for measuring differential absorption. There were 122 tuning frequencies in the sweep, separated by $0.25 \mathrm{GHz}$, except for a gap between 191.5 and $193.5 \mathrm{GHz}$. This gap is due to the limited receiver response for intermediate frequencies (IF) less than $1 \mathrm{GHz}$ and the first-stage local oscillator (LO) being set to $192.5 \mathrm{GHz}$.

When executing the tuning sequence, the tuned transmitter tone dwelled at a particular frequency in the tuning sequence for $100 \mathrm{~ms}$ before moving to the next frequency in the sequence. The timing of the transmitter-receiver tuning was synchronized using GPS receivers. Each received ATOMMS signal was filtered, down converted in frequency, digitized and recorded. The signal frequency in the final receiver stage ranged from 8 to $35 \mathrm{kHz}$ for each of the 122 tuned frequencies. The frequency and power of the down-converted signals were determined using a fast Fourier transform (FFT), calculated over a $50 \mathrm{~ms}$ integration time. The reason that only half of the $100 \mathrm{~ms}$ tuning dwell time was used was to allow time for each synthesizer tune to settle. Each FFT-derived signal power estimate was then converted to an amplitude by taking the square root. The calibration signal amplitudes were computed using the same method.

One sweep through the frequency tuning sequence took $12.2 \mathrm{~s}$. The instrument cycled through the four combinations of the two transmitters and two receivers before repeating the tuning cycle in order to help isolate any transmitter or receiver issues. Thus, a full tuning cycle was completed every $48.8 \mathrm{~s}$. The observations from the four combinations of transmitter-receiver pairs were then averaged together such that new estimates for the ATOMMS signal amplitude ratios at all of the 122 tuning frequencies were generated every $48.8 \mathrm{~s}$ (Eq. 2). As a result, the integration time used to estimate the signal amplitude and frequency for each of the 122 frequencies in the tuning sequence was 4 times 50 or $200 \mathrm{~ms}$.

\section{Interpretation of measurements}

ATOMMS observations of $R$, defined in Eq. (2), are sensitive to changes in the integrated water vapor along the path between the instruments. The retrieval algorithm discussed below determines changes in water vapor pressure relative to a reference period. We selected two reference periods that are identified in Fig. 2. The first period spanned 14:23 to 14:31, shortly after data acquisition began, and the second spanned $16: 51$ to $16: 56$, approximately $2.5 \mathrm{~h}$ later. These are periods of relatively constant amplitude spectra due to relatively constant vapor pressure and temperature and relatively low optical depth, which maximizes the number of usable frequencies nearest the line center. Comparing solutions de- 
rived using the two different reference periods provides some assessment of instrumental drift.

The retrieval algorithm determines the change in vapor pressure relative to the reference period by finding the best forward-calculated fit to each observed ATOMMS amplitude ratio spectrum (Eq. 2) using a least squares method. To forward model the clear-sky atmospheric attenuation, we used an atmospheric propagation tool known as the atmospheric model (am), version 7.2 (Paine, 2011), which we will refer to as am7.2. This model was shown to fit the ATOMMS measurements to the $0.3 \%$ level in previous work with the ground-based ATOMMS prototype system (Kursinski et al., 2012). In operation, the ATOMMS ratio, $R$ in Eq. (2), is determined from measurements at times, $t$ and $t_{0}$, for a range of frequencies, $f$, which produces a frequency spectrum of the ratio. In forward calculations of Eq. (2), we assume that the vapor pressure, air temperature and air pressure are known at the reference time, $t_{0}$, and the air pressure and temperature are known at time, $t$. The solution is determined by finding the change in vapor pressure from the reference value that provides the best least squares fit between the forwardcalculated and observed ATOMMS ratio spectra. During this experiment, we were able to accurately determine signal amplitudes up to total optical depths due to gas plus liquid water of 17.

For the purposes of determining the average water vapor along the path, we used 15 tuning frequencies spanning 187.861 to $191.361 \mathrm{GHz}$ for the water vapor retrievals. Since the greatest sensitivity to changes in vapor pressure occurs at the line center, it is desirable to utilize frequencies as close to the line center as possible. For this field test, tuning tones with frequencies lower than $187.861 \mathrm{GHz}$ were too attenuated to be measured accurately even during clear skies. During periods of lighter rain and clouds, the additional attenuation by liquid water caused the retrieval frequencies nearest the line center to become too opaque to be measured accurately, reducing the number of frequencies available for the fit. The liquid optical depth in Fig. 2 is the liquid optical depth measured by the calibration signal, $f_{\mathrm{CAL}}=$ 198.5 GHz. The liquid optical depth was computed by subtracting the forward-calculated change in gaseous extinction relative to the reference period from the observed change in optical depth relative to the reference period, which includes changes in both liquid and gaseous extinction. During the heaviest rain period, none of the high-band signals could be measured due to strong liquid attenuation.

The retrieved path-averaged vapor pressure between the instruments is shown in Fig. 3a. The figure shows 12 different solutions that were used to estimate the random uncertainty in the retrieval of vapor pressure. The methodology used to compute the 12 solutions is described in Sect. 5. The half range of the 12 solutions shown in Fig. $3 \mathrm{~b}$ is generally less than $0.1 \mathrm{hPa}$. Most of the fractional uncertainties are well below $1 \%$ of the vapor pressure, indicating that the solution is highly constrained by the observations. The path-averaged vapor pressure varied from 10.2 to $16.5 \mathrm{hPa}$ over the nearly $4 \mathrm{~h}$ observation period. The measured vapor pressure peaked in association with the rainy period before 15:00. Following that rain shower, there was a brief intrusion of drier air centered near 15:15 before the vapor pressure rapidly increased prior to the thunderstorm at 15:30. Immediately following the heavy rain after reacquisition of the high-band signals, the vapor pressure dropped to its lowest value. In Sect. 6, we note that similar advection of dry air following summertime thunderstorms in this region have been observed in previously published work (Kursinski et al., 2008) and show that our estimation of the minimum vapor pressure was consistent with the nearby radiosonde observations from Tucson. During the brief cloud passage at 16:30, there was a sharp increase and peak in the vapor pressure that brought the relative humidity up to approximately $100 \%$. The vapor pressure fell sharply following the passage of the cloud. There was one more peak in vapor pressure at 17:00 before the sharp rise associated with the rain that began at 17:30.

\subsection{Determining temperature}

Retrieving changes in water vapor vs. time from the measured absorption spectra requires knowledge of atmospheric temperature and pressure. In the eventual LEO-LEO occultation measurements, ATOMMS will profile both the atmospheric Doppler shift and attenuation of the occulted signals, from which profiles of temperature, pressure and water vapor will be derived (Kursinski et al., 2002). In the static mountaintop-to-mountaintop geometry, there is no Doppler shift and only the attenuation portion of the ATOMMS measurements is available. Pressure was determined using barometers on each mountaintop. Determining the atmospheric temperature along the signal path was more challenging.

During this experiment, three nearby thermometers measured the surface air temperature. An Arduino weather station was located next to each ATOMMS instrument and an automated weather station was located in the town of Summerhaven, about $300 \mathrm{~m}$ below Mt. Lemmon and $700 \mathrm{~m}$ to the north. Unfortunately, these surface temperature observations were not entirely representative of the air temperature aloft along the ATOMMS signal path because of their close proximity to the surface and a high bias in the Arduino temperatures due to heat generated by the ATOMMS instrumentation inside the protective tents.

To better estimate the temperature along the signal path, we derived the average air temperature along the path from the pressure scale height using the hypsometric equation and time-varying barometric pressure measured at the two ATOMMS instruments: 

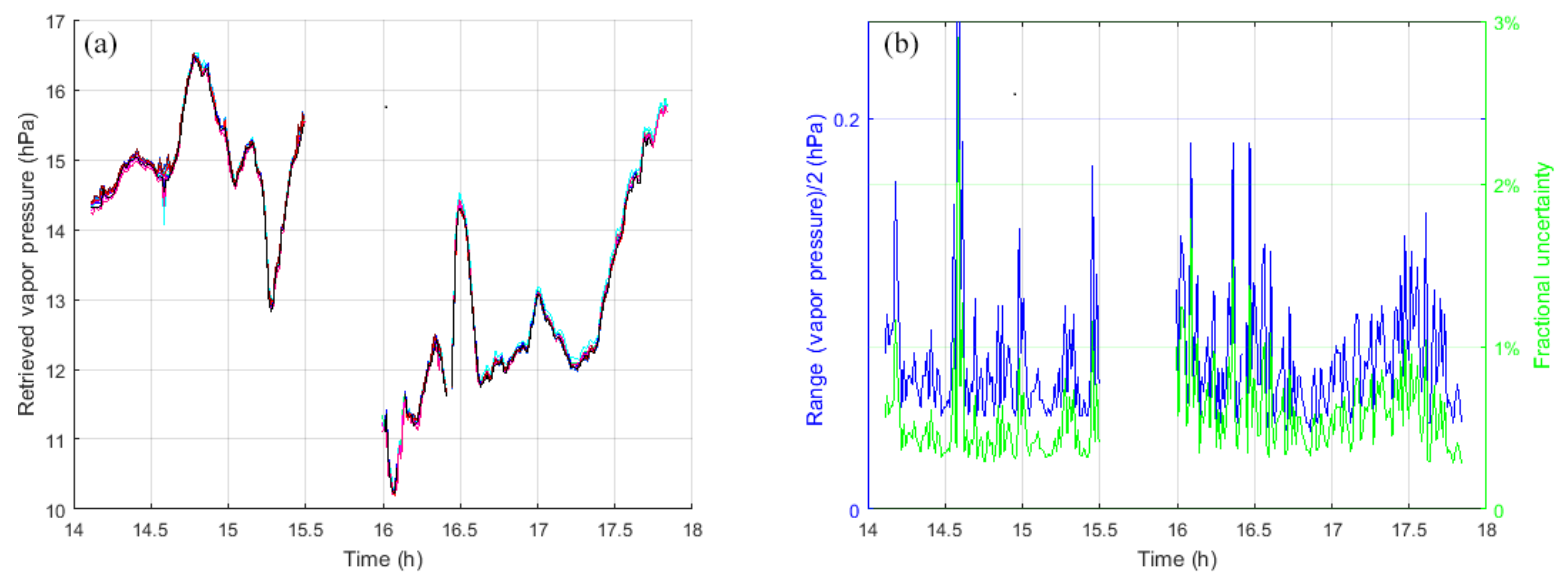

Figure 3. (a) Retrieved vapor pressure for the 12 retrieval test cases described in the text. Each line is a different color. (b) Blue line and left axis indicate the half range, which is half of the maximum minus minimum vapor pressure from the 12 retrieval cases; green line and right $y$ axis is the half range divided by the absolute vapor pressure at each retrieval point expressed in percent. The strong peaks near $14.6 \mathrm{~h}$ are due to momentary noise in the calibration signal.

$\overline{T_{\mathrm{V}}}=\frac{g \Delta Z}{R_{\mathrm{d}}}\left[\ln \left(\frac{P_{\mathrm{Big}}}{P_{\mathrm{Lem}}}\right)\right]^{-1}$

where $g$ is gravitational acceleration, $\Delta Z$ is the altitude difference between Mt. Lemmon and Mt. Bigelow, $R_{\mathrm{d}}$ is the gas constant for dry air, $P_{\text {Big }}$ and $P_{\text {Lem }}$ are the measured air pressures on Mt. Bigelow and Mt. Lemmon respectively, and $\overline{T_{\mathrm{V}}}$ is the layer mean virtual temperature. The air temperature is obtained from the virtual temperature, e.g., Wallace and Hobbs (1977).

While Eq. (5) ideally provides the desired layer mean temperature needed for spectral calculations of $R$, there are issues with this approach. The sensitivity of Eq. (5) to small dynamic pressure variations made short-term temperature estimates noisy. The horizontal separation between Mt. Lemmon and Mt. Bigelow caused the estimated temperature to be sensitive to propagating pressure perturbations. Finally, the assumption of hydrostatic balance in Eq. (5) is not true during thunderstorm activity. To alleviate these issues, we used a $1 \mathrm{~h}$ running mean of the air pressure.

Temperatures derived in this manner are biased by small biases in barometric pressure. To minimize this bias, we shifted the entire temperature time series by $2.15 \mathrm{~K}$ so that the relative humidity was $100 \%$ at $16: 30$, when the cloud was present. Figure 4 shows the derived air temperature between the instruments that was used in the retrievals in black, as well as the nearby in situ thermometer observations, which are shown in red, green and blue. The uncertainty associated with this temperature estimation is discussed in Sect. 5.

\subsection{Water vapor spectra}

Figure 5 shows four examples of fitted ATOMMS ratio spectra. The outstanding agreement between the measured and

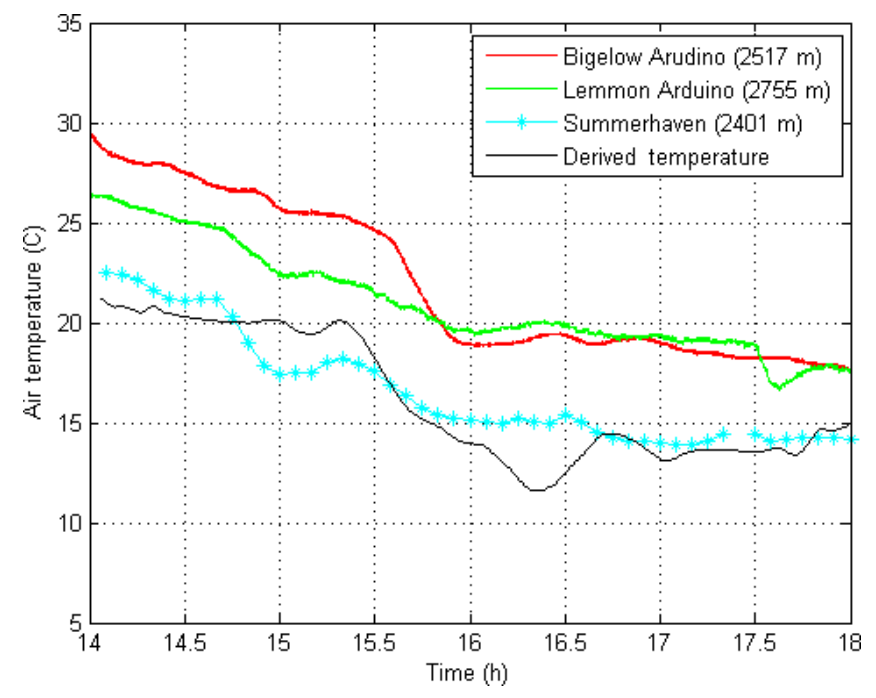

Figure 4. Observed and derived air temperatures during the ATOMMS ground-based experiment.

modeled spectra is immediately evident, in that most of the individual ATOMMS amplitude ratio spectra fall within $\pm 0.15 \mathrm{hPa}$ (which is $\pm 1 \%$ ) of the calculated spectra. This is true for most of the individual retrievals.

Figure 5a shows a retrieval made during the clear period around 15:08, following the first rain period. All 15 frequencies spanning 187.861 to $191.361 \mathrm{GHz}$ were available and closely fit the forward-calculated ATOMMS ratio. Figure 5b shows a retrieval made during the first rain period at 14:51. While the two frequencies nearest the line center were lost due to the increase in optical depth caused by rain, the remaining 13 ATOMMS frequencies yielded accurate vapor pressure retrievals during the rain. 

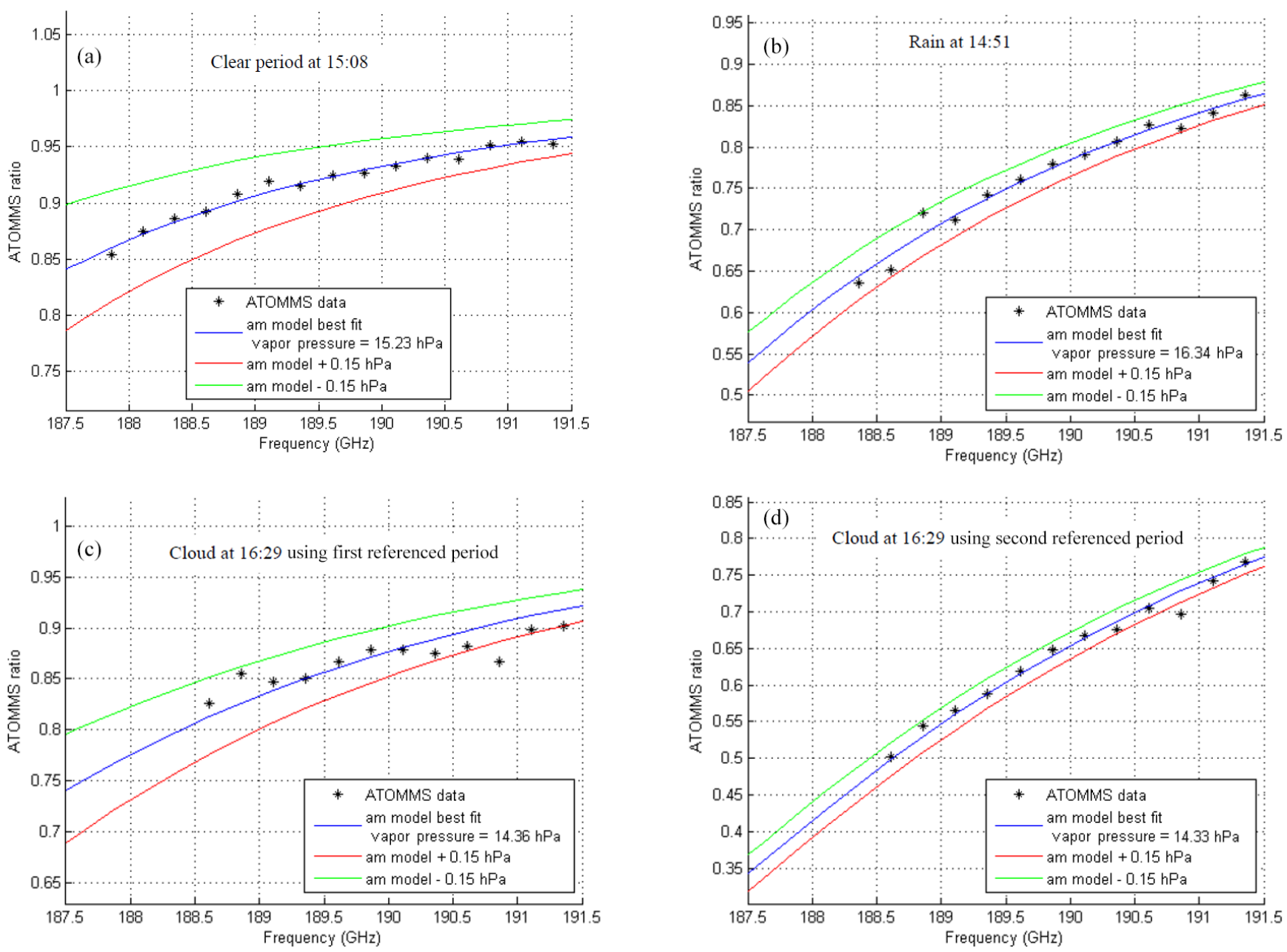

Figure 5. Examples of fitting the observed ATOMMS amplitude ratio, Eq. (2) (black asterisks), to the forward calculated ATOMMS ratio using am7.2. Blue line is the best-fit line for the indicated vapor pressure. Red line is am forward calculation for a vapor pressure $0.15 \mathrm{hPa}$ greater than the best-fit vapor pressure. Green line is forward calculation for a vapor pressure $0.15 \mathrm{hPa}$ less than the best-fit vapor pressure. The solutions shown in panels (a), (b) and (c) used reference period 1, while the solution in (d) used reference period 2.

Figure 5c and d show retrievals made at 16:29, during the cloudy period. The solution in Fig. $5 \mathrm{c}$ uses the first reference period, while the solution in Fig. 5d uses the second reference period, which is closer to the time of the cloudy period. The difference between the shapes in the ATOMMS ratio spectrum in Fig. $5 \mathrm{c}$ and $\mathrm{d}$ is due to the use of the two different reference periods, which change the amplitude ratio in the denominator of Eq. (2). The increased liquid optical depth due to the cloud eliminated the three frequencies nearest the line center. Although scatter about the best-fit forward calculation line is larger than that in Fig. 5a and b, the fitted forward calculations constrain the water vapor solution quite well, despite the presence of the cloud and some light rain. The better fit that results when using the second reference period indicates that there was some subtle instrumental drift over the $2.5 \mathrm{~h}$ between reference periods. Near the cloud peak, the Reference 1 water vapor solutions are greater than the Reference 2 solutions by only $0.03 \mathrm{hPa}(0.2 \%)$, indicating the level of robustness of these vapor pressure retrievals.

\section{Sources of uncertainty and validation of results}

There are a number of sources of uncertainty in the ATOMMS mountaintop water vapor retrievals that include

1. measurement errors including signal-to-noise-ratio (SNR) and instrument drift,

2. undesired environmental effects such as scintillations due to turbulence,

3. errors in modeling including gaseous spectroscopy and particulate scattering,

4. biases due to errors in the reference period air temperature and water vapor estimates, 
5. errors in the estimated time-varying, path-averaged, air temperature, and

6. uncertainty in spectral fitting.

In terms of measurement errors (Category 1), the high SNR that enabled penetration and water vapor retrievals up to optical depths of 17 is not a significant source of error, except, of course, when optical depths exceeded 17 and became impenetrable. As noted, we did see signs of subtle instrument drift over approximately $2.5 \mathrm{~h}$, which is $9000 \mathrm{~s}$, which shifted the retrieved water vapor amount by $0.2 \%$. However, because the duration of a LEO occultation is only about $100 \mathrm{~s}$, errors due to instrument drift in LEO should be very small.

Turbulence-induced amplitude scintillations (Category 2) were quite significant during the periods of strong convection. These were reduced by almost an order of magnitude via amplitude ratioing with the calibration signal (Kursinski et al., 2016b). The strong peaks near $14.6 \mathrm{~h}$ in Fig. $3 \mathrm{~b}$ are caused by momentary noise in the calibration signal, which influences the frequency ratioing. Outside of this peak the largest fractional uncertainty is about $1.8 \%$ of the vapor pressure (green line). We attribute most of this to turbulentinduced scintillations that remain after the frequency ratioing. Thus, for the conditions of this field experiment, the upper bound for the random error in the vapor pressure retrieval due to turbulence is about $1.8 \%$ of the vapor pressure.

In terms of spectroscopic errors (Category 3), we again note that ATOMMS is itself a very high spectral resolution spectrometer such that the ATOMMS data can be used to refine the spectroscopic models and make them as accurate as the ATOMMS observations. Along these lines, we also note that, in order to diagnose and reduce spectroscopic errors, Kursinski et al. (2009) recommended increasing the required number of low-band signals from 4 to 5 to make the solutions systematically overdetermined in order to identify systematic errors in spectroscopic models and then refine those models.

Errors in the reference period temperature and water vapor estimates (Category 4) create unknown biases in our mountaintop estimates. These biases are not relevant to the eventual LEO system because, in the LEO-LEO occultation geometry, the reference period occurs when the signal path is above the detectable atmosphere, where the atmospheric density is essentially zero.

The primary cause of temperature-related uncertainty is in the change in temperature between the reference period and the observation time (category 5). Errors in the absolute temperature are relatively insignificant; i.e., temperature biases are not a significant source of uncertainty in the water vapor retrievals in comparison to errors in estimating the change in temperature relative to the reference periods. For the conditions of this particular experiment, based on forward calculations made with am7.2 for the range of temperature and vapor pressure conditions observed during the experiment, the sensitivity of the change in derived water vapor due to a temperature change relative to the reference period temperature

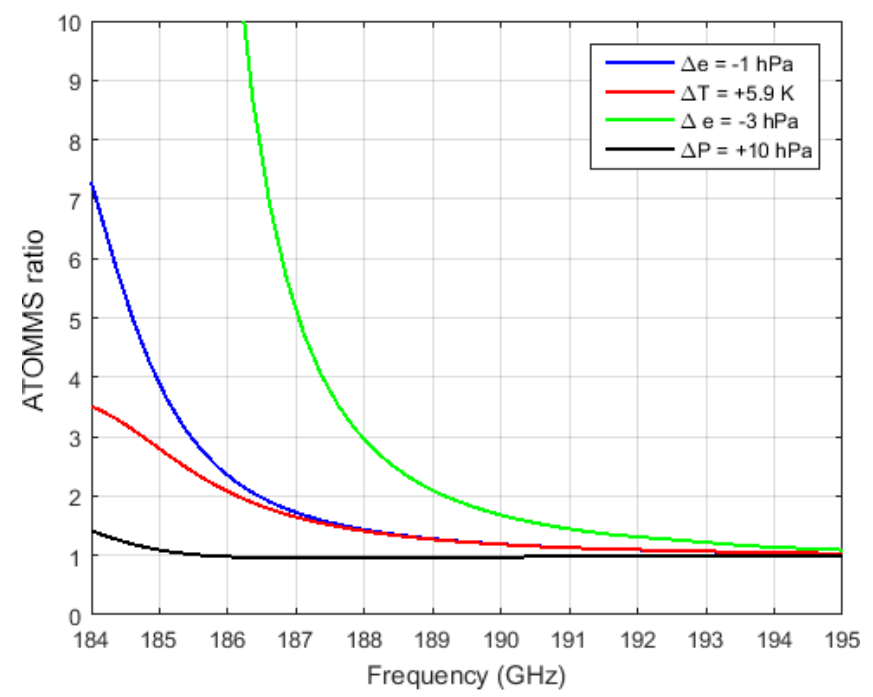

Figure 6. ATOMMS ratio for four changes in the atmospheric conditions along the $5.4 \mathrm{~km}$ observation path relative to reference conditions: vapor pressure decreased by $1 \mathrm{hPa}$ (blue), temperature increased by $5.9 \mathrm{~K}$ (red), vapor pressure decreased by $3 \mathrm{hPa}$ (green) and air pressure increased by $10 \mathrm{hPa}$ (black). The reference conditions were air pressure $=743 \mathrm{hPa}$, air temperature $=20^{\circ} \mathrm{C}$ and vapor pressure $=15 \mathrm{hPa}$.

was approximately $-0.17 \mathrm{hPa}^{\circ} \mathrm{C}^{-1}$. Examples of the sensitivity of the ATOMMS ratio, Eq. (2), to changes in vapor pressure, temperature and air pressure relative to the reference conditions for this experiment are shown in Fig. 6. The figure plots the forward-computed ATOMMS ratio spectrum for four different changes relative to the reference conditions. For the conditions of the field experiment, we were able to measure amplitudes for signal frequencies of $187.861 \mathrm{GHz}$ and higher. Lower frequencies closer to the line center were too attenuated to track. The figure shows the change in the ATOMMS ratio spectrum resulting from a change in air pressure of $10 \mathrm{hPa}$, which is much larger than the $\pm 2 \mathrm{hPa}$ changes in air pressure that were observed during the experiment. Therefore, the sensitivity of the ATOMMS ratio to changes in air pressure is quite small relative to changes in vapor pressure. As the figure shows, for frequencies greater than $187.861 \mathrm{GHz}$, a $1 \mathrm{hPa}$ decrease in vapor pressure produced approximately the same ATOMMS amplitude ratio spectrum as a $5.9^{\circ} \mathrm{C}$ increase in air temperature. Larger changes in vapor pressure, such as the $-3 \mathrm{hPa}$ line in the figure, are easily distinguished from changes in air temperature. Based on Fig. 4, the uncertainty in the change in temperature relative to the reference period temperature during this experiment was less than $3{ }^{\circ} \mathrm{C}$, which places an upper bound of a $0.5 \mathrm{hPa}$ water vapor uncertainty due to the temperature uncertainty.

The misfit between the measured ATOMMS amplitude spectral ratios and the forward calculation of those spectral ratios (category 6) are sensitive to all of the error types noted above. To understand and characterize the robustness 
in the spectral fits, we varied the number of frequencies used in the fits. The baseline retrieval utilized the amplitudes of the 15 signals with frequencies ranging from 187.861 to 191.361 GHz. Five additional retrievals were implemented using different subsets of these 15 frequencies. Specifically these subsets were the 10 lowest frequencies, the 10 highest frequencies, the five lowest frequencies, the five middle frequencies and the five highest frequencies within the 187.861 to $191.361 \mathrm{GHz}$ frequency range. We also ran the same six cases using the second reference period. The same temperature vs. time was used for all 12 cases.

Figure 3 a shows the resulting 12 solutions. The blue line in Fig. $3 \mathrm{~b}$ shows the spread across the 12 retrievals, defined as the maximum minus the minimum vapor pressure divided by two. This half range represents a conservative estimate of the random uncertainty of the retrieved vapor pressure changes that include both measurement and am7.2 modeling errors. The average half range is $0.077 \mathrm{hPa}$, which corresponds to a fractional uncertainty of approximately $0.6 \%$. This small spread across the 12 cases indicates that instrument drift over the $4 \mathrm{~h}$ observational period was quite small and that the ATOMMS spectral observations tightly constrained the vapor pressure with little ambiguity over a wide range of clear, cloudy and rainy conditions in optical depths up to 17 .

The amplitude ratio in Eq. (2) reduces common-mode sources of error and uncertainty. Ratioing of the amplitudes of two signals, as was done here, eliminates the effects of liquid particle extinction to the extent that the liquid extinction is spectrally flat over the ATOMMS tuning range and calibration frequencies. For raindrop-sized spheres of water, Mie theory predicts that the millimeter wavelength spectrum of extinction is nearly flat. For smaller cloud droplets, Mie theory combined with the dielectric model of liquid water indicates that the millimeter (and centimeter) wavelength extinction increases approximately linearly with frequency due to absorption by liquid water. Near 16:30, the passage of a cloud between the mountaintops coincided with an increase in the $198.5 \mathrm{GHz}$ extinction but no increase in the $24.4 \mathrm{GHz}$ extinction, indicating the presence of very small particles along the path. We adjusted the retrieval algorithm to account for this expected cloud droplet spectral dependence over the high-band frequency range, which caused the retrieved vapor pressure to increase by $0.8 \%$. The increase was necessary to compensate for the slight spectral variation in liquid water attenuation that resulted from using the Mie cloud model (Bohren and Huffman, 1983). Surprisingly, the spectral misfit to the ATOMMS observations increased slightly. The reason is not clear.

This small $0.8 \%$ change in the retrieved vapor pressure provides some indication of how effective the calibration signal ratioing is in minimizing the sensitivity of the ATOMMS water vapor retrievals to hydrometeors. In the future, the high-band system will have four rather than its present two signals in order to place calibration signals on both the lowand high-frequency sides of the $183 \mathrm{GHz}$ water vapor line to reveal and compensate for any overall spectral tilt caused by particle extinction as well as other effects. This should greatly reduce cloud ambiguity in the $183 \mathrm{GHz}$ based water vapor retrievals.

\section{Validation against in situ measurements}

In previously published work, we demonstrated the ability of the ATOMMS prototype system to accurately retrieve changes in water vapor along a relatively short $820 \mathrm{~m}$ path across the University of Arizona campus in clear conditions. In that experiment, the atmosphere was well mixed and nearly homogeneous along the observation path such that the retrieved changes in water vapor from ATOMMS matched those observed with an in situ sensor near one end of the path to $1 \%-2 \%$ (Kursinski et al., 2012). Based on these results, our intent had been to validate these ATOMMS moisture retrievals in the presence of clouds and rain via comparison with independent, in situ moisture measurements analogous to the $\sim 1 \%$ validation of clear-sky ATOMMS retrievals along a shorter path demonstrated by Kursinski et al. (2012). However, we came to realize that quantitative validation of the ATOMMS water vapor retrievals for this mountaintop experiment was limited by the substantial spatial inhomogeneity of the moisture field itself associated with a longer path, over mountainous terrain, during thunderstorm activity. The large variations in water vapor produced by the turbulent, moist, convective activity limited the level of agreement between several in situ sensors.

The spatial inhomogeneity of the water vapor field is evident in Fig. 7, which shows ATOMMS water vapor retrieval and observations from three nearby in situ sensors as well as the measurement from the Tucson radiosonde at the altitude of the ATOMMS experiment. The differences between the in situ sensors are indicative of the magnitude of moisture variations along the $5.4 \mathrm{~km}$ path. The observation geometry in Fig. 1 shows that the ATOMMS-derived vapor pressure is an average over the $5.4 \mathrm{~km}$ path that runs above a valley between the mountaintops on which the instruments sit. The high-band transmitter was located at the position marked and labeled "Physics/Atmos bldg Radio Ridge" at an altitude of $2752 \mathrm{~m}$ and the high-band receiver was located at the position marked and labeled "Catalina Station Steward Observatory" at an altitude of $2515 \mathrm{~m}$. In situ sensors were located on the ground at the two instrument sites, with another at the location marked and labeled "Summerhaven", which is about $830 \mathrm{~m}$ from the observation path in a valley at an elevation of $2439 \mathrm{~m}$.

The spatial variability of the water vapor during this experiment was large. A measure of the water vapor variability over the $5.4 \mathrm{~km}$ observation path is provided by computing the root mean square (rms) differences for the three available in situ sensors during the experiment, namely the two sensors at each end of the observation path and data from a sensor in 


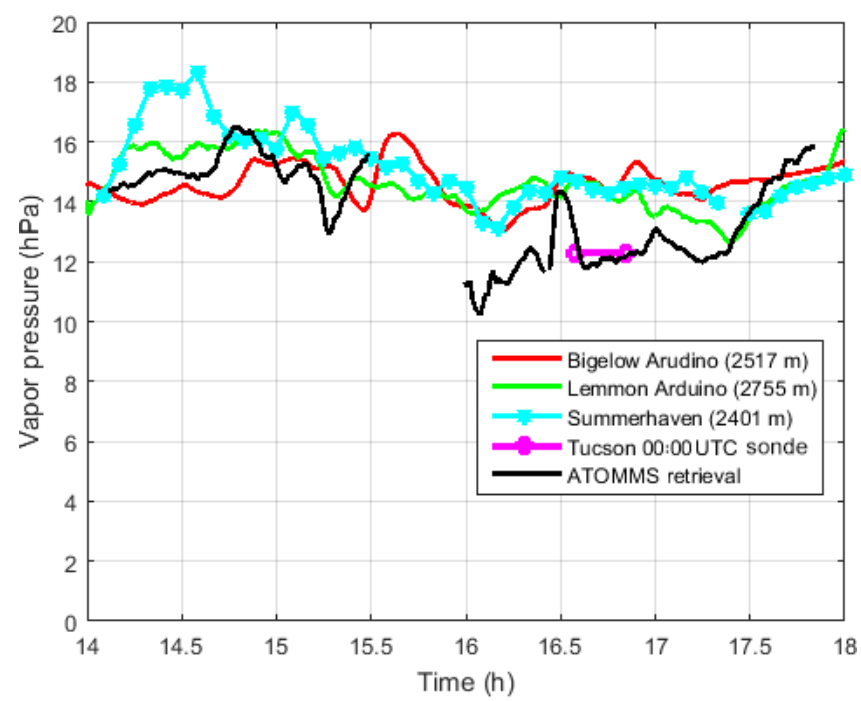

Figure 7. Observed and retrieved vapor pressures. The sonde line indicates the average vapor pressure over the altitude range of the ATOMMS instruments as reported in the 00:00 UTC Tucson sonde for 19 August.

the town of Summerhaven in the valley below the observation path. The rms of the differences between the three in situ sensors and the ATOMMS-derived water vapor was approximately $8 \%$ during the period from $14: 00$ to $15: 30$, which preceded the first heavy rain period. Water vapor variations during the most active convective periods were likely larger. In the appendix, we discuss the difficulty and very high (prohibitive?) cost of designing and employing an in situ observational network capable of verifying the ATOMMS retrievals for the conditions encountered during this experiment.

\subsection{Cross correlations}

Despite the inherent differences in the horizontal averaging of ATOMMS and the in situ instruments, there is substantial cross-correlation between these water vapor measurements. We show this by examining the correlation between the ATOMMS-retrieved path-average water vapor and the in situ water vapor sensor located on Mt. Bigelow. Figure 8 a shows the ATOMMS retrieval for the path-averaged vapor pressure in blue and the measured vapor pressure from the in situ sensor on Mt. Bigelow in red. Substantial crosscorrelation is clearly evident between the two data sets. The other colored lines in Fig. 8a show time-shifted segments of the in situ observations, as described below, that make the correlation between the data sets more visually apparent. In order to demonstrate and quantify the cross-correlation between the ATOMMS-derived vapor pressure and the in situ observations, we separated the data sets into several different time segments because the time lag between the two observations of water vapor varies as the wind conditions change. We discuss four particular time segments defined as follows
1. 14.06 to $14.79 \mathrm{~h}$, which is approximately the first $45 \mathrm{~min}$ of data collection;

2. 14.99 to $15.49 \mathrm{~h}$, which is the period leading up to the first heavy rain period when the ATOMMS high-band signals became too attenuated to track;

3. 16.00 to $16.42 \mathrm{~h}$, which is the period when the highband signals reappeared following the heavy rain; and

4. 16.75 to $17.39 \mathrm{~h}$, which is the period immediately following the cloudy period.

Figure $8 \mathrm{~b}$ shows the correlation coefficients as a function of sample time lag. Consecutive ATOMMS samples are separated by $48.8 \mathrm{~s}$. The peak cross-correlation coefficients range from 0.78 to 0.97 , which indicate strong correlation between the ATOMMS-derived water vapor pressure and the in situ observations of water vapor pressure on Mt. Bigelow. Positive lags indicate periods when ATOMMS-observed water vapor variations occurred earlier than those variations in the in situ observations on Mt. Bigelow. Although the winds were occasionally gusty, with variable direction due to shower and thunderstorm activity, there were two systematic shifts in the prevailing wind direction observed in the field: a shift from W to NNW around 15:48 and a shift from NNW to ENE around 16:55. These wind shifts were observed both from the motion of clouds in sequences of web camera images taken from Mt. Bigelow and by the Tucson WSR-88D radar (Crum and Alberty, 1993). The ATOMMS instruments were oriented along a NE-to-SW direction, with Mt. Bigelow on the SW end (Fig. 1). Figure $8 \mathrm{~b}$ indicates that the first three time segments had positive lags, while the last time segment had a negative lag. This is consistent with our wind observations, in which the wind direction had a component from the observation path toward Mt. Bigelow for the first three time periods, and from Mt. Bigelow to the observation path for the fourth time period.

\subsection{Moist bias in in situ sensor sampling}

Another issue in validating the ATOMMS water vapor retrievals against the in situ sensor results is a moist bias in the ground measurements relative to the overlying air after the period of heavy rain. The bias is due to evaporation from the wet surface moistening the near-surface air, which is the air with properties that are measured by the in situ sensors. As a result, with the exception of the cloud around 16:30, the retrieved ATOMMS water vapor amounts over the 80 min following the heavy rain were systematically lower than the surface measurements. This continued until approximately 17:20, when the steady increase in water vapor and rain began and continued until the end of the experiment. The largest differences occurred shortly after the most intense rain, when ATOMMS measured a vapor pressure of $10.2 \mathrm{hPa}$, the smallest of the entire experiment. This value is approximately $25 \%$ lower than water vapor measured at the 

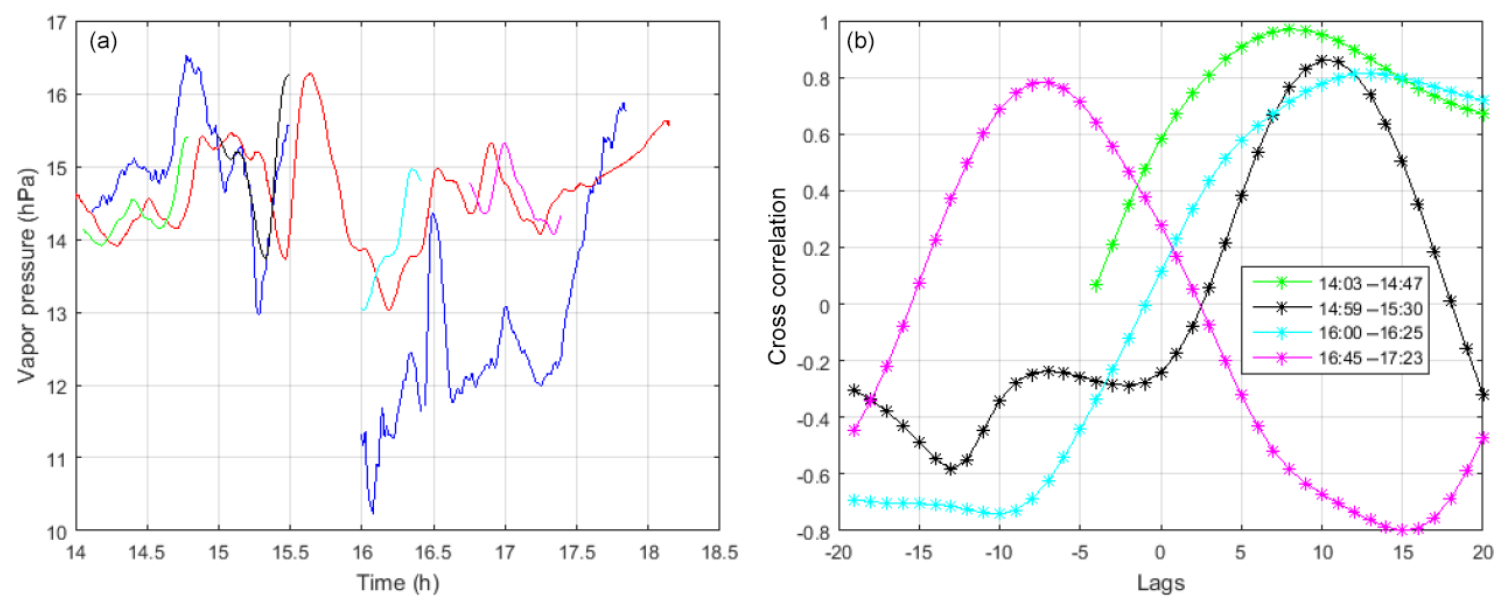

Figure 8. (a) Vapor pressure derived from ATOMMS observations (blue) and measured with an in situ sensor on Mt. Bigelow (red). Also shown in other colors are four time segments of the in situ observations shifted in time (as described in the text) to highlight the correlation between the two vapor pressure data sets. The time shift for each colored line is indicated in (b). (b) Cross-correlation coefficients as a function of sample lags between the ATOMMS-derived vapor pressure and in situ measurements of water vapor taken on Mt. Bigelow. The four lines correspond with the four time segments described in the text: green (14:03-14.47), black (14:59 to 15:30), cyan (16:00 to 16:25) and magenta (16:45 to $17: 23)$.

surface stations. Such behavior where moisture at the surface varies little, while air aloft becoming significantly drier following summertime thunderstorms is common in this region (e.g., Fig. 4 in Kursinski et al., 2008). It is also common in the Amazon (e.g., Fig. 7 in Schiro et al., 2016) and may be associated with mid-level inflow of drier air into the precipitating region that results in evaporative cooling and descent of this air (e.g., Leary, 1980; Houze, 2004).

For the period of relatively dry air following the cloud, the 00:00 UTC Tucson radiosonde profile provides perhaps the best validation of the ATOMMS results. The sonde was launched between 16:30 and 16:45 from a location about $28 \mathrm{~km}$ southwest of the experiment and ascended through the Mt. Bigelow to Mt. Lemmon altitude interval between $16: 35$ and $16: 50$ at a location approximately $20 \mathrm{~km}$ south of the observation path. According to the sonde, the average vapor pressure in the layer between Mt. Bigelow and Mt. Lemmon was about $12.3 \mathrm{hPa}$, which is within a few percent of the ATOMMS water vapor retrievals following the cloud's passage. We also note that moisture concentrations measured on Mt. Lemmon decreased steadily through this period, reaching a minimum of $12.7 \mathrm{hPa}$ at $17: 25$, a value essentially identical to the ATOMMS moisture retrieval at this time (Fig. 7). This decrease, despite the evaporative moistening from the wet surface, suggests that dry air was indeed advecting over Mt. Lemmon. Thus, the combination of the sonde profile, the ATOMMS measurements and Mt. Lemmon surface measurements all indicate passage of a relatively dry, horizontally extended air layer following the heavy rain.

Further examination of the operational sonde profiles launched in Tucson that morning around 04:30 and that afternoon, around 16:30, provide additional clues as to what hap- pened that afternoon. Figure 9 shows the specific humidity and potential temperature calculated from the Tucson $19 \mathrm{Au}-$ gust, 00:00 UTC sonde for the lowest $3000 \mathrm{~m}$ above Tucson. The green hatched region shows the altitude interval across the ATOMMS observation path. In the afternoon sonde profile, the potential temperature, $\theta$ and specific humidity, $q$, are nearly constant between the surface and $2300 \mathrm{~m}$ above sea level (m.s.l.), indicating that the boundary layer (BL) near 16:30 local time extended to about 2300 m.s.l. In contrast, cloud base at 3150 m.s.l., where the dew point equals the temperature in the sonde profile, and the $500 \mathrm{~m}$ nearadiabatic layer immediately below it, further indicate that earlier in the afternoon, the well-mixed, dry adiabatic subcloud BL very probably extended up to $3150 \mathrm{~m}$.s.l. Between 2300 and 2750 m.s.l. is a thermal inversion layer that is noticeably drier than the air immediately above and below it. The ATOMMS measurements were made within this altitude interval. The relatively low moisture concentrations in this layer measured by both ATOMMS and the afternoon sonde, combined with the fact that the $\theta$ of this inversion layer is lower than the $\theta$ of the peak afternoon BL indicates that this air was likely cooled diabatically by evaporation of precipitation falling through it during the turbulent period of heavy rain. The net effect of this process was to increase the $q$ and reduce the $\theta$ of this air, causing it to descend from a higher altitude to where it was measured by ATOMMS. Similarly, the fact that the $\theta$ of the late afternoon boundary layer below the ATOMMS layer is $2.5 \mathrm{~K}$ lower than that of the peak afternoon BL also indicates that that air has also been evaporative cooled and descended as a result. Such evaporative cooling and descent and moistening of dry-air layers is a well-known feature of squall lines (e.g., Houze, 2004) and 


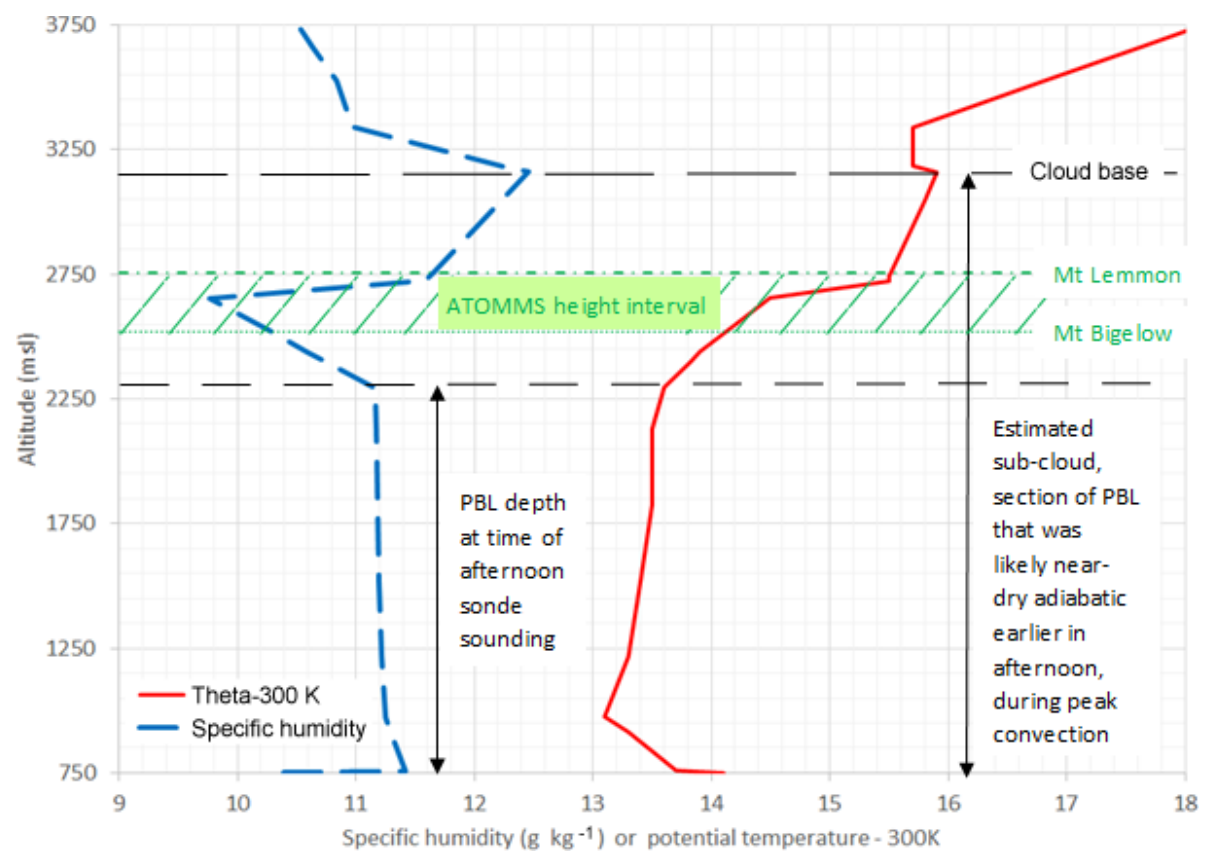

Figure 9. Vertical profiles of specific humidity and potential temperature minus $300 \mathrm{~K}$ calculated from the 00:00 UTC Tucson sonde. The local time of the sonde launch was approximately 16:30 on 18 August. Theta label for the red line stands for potential temperature and PBL stands for planetary boundary layer.

causes microbursts, which are well known in Arizona (e.g., Willingham et al., 2010). Further understanding of the details of what happened that afternoon will require detailed modeling with a convection-resolving model, which is beyond the scope of the present research.

\section{Discussion}

The results of this ATOMMS field test demonstrate that the differential absorption concept using an active microwave spectrometer works very well, yielding performance consistent with theoretical expectations that is well beyond the capabilities and performance of passive radiometers. Using a prototype ATOMMS instrument we developed, we measured differential absorption spectra and then forward modeled those spectra, achieving better than $1 \%$ agreement, through clear air, clouds and rain to determine the changes in the pathaveraged water vapor pressure between the ATOMMS instruments. We demonstrated water vapor retrievals made during cloudy and rainy periods that were only slightly noisier than those made during clear-sky periods. Accurate retrievals of water vapor pressure were made through optical depths up to 17 , thus demonstrating the exceptionally wide dynamic range achievable via the differential absorption approach. The fact that this performance was achieved under turbulent conditions associated with intense, local thunderstorms also indicates the effectiveness of the differential approach in reducing the impact of turbulence.
While the variable, turbulent conditions associated with convective activity together with passing clouds and rain provided an excellent test of the ATOMMS system's ability to function and perform in very challenging conditions, it also limited the level of validation that could be achieved against in situ surface sensors. The disagreement amongst the three nearby in situ sensors revealed the substantial inhomogeneity in the water vapor field in the vicinity of the $5.4 \mathrm{~km}$ observation path. Prior to the first heavy rain period, the rms of the differences between the in situ sensors was approximately $8 \%$, which set an upper bound to which the ATOMMS-retrieved changes in water vapor pressure could be validated by the in situ sensors. It is also important to note that ATOMMS measured the change in the path-averaged vapor pressure, which will differ somewhat from point measurements along the path with a magnitude that depends on the inhomogeneity of the water vapor along the path.

During the period following the heavy rain, the ATOMMS measurements revealed systematically drier conditions than the nearby in situ sensors. These differences were likely due to the fact that the in situ sensors were located at the surface, while the path between the ATOMMS instruments was aloft. As a result, the in situ sensors measured the humidity of air moistened by evaporation from the rain-soaked surface, while ATOMMS measured the humidity of air aloft the valley between the two instruments. The nearby Tucson radiosonde indeed indicated that, following the thunderstorm, a layer of drier air passed through the area. Thus, direct validation of the ATOMMS retrievals against the in situ sensors 
was limited to about $8 \%$. In the appendix we discuss why it would have been extremely difficult to validate our retrievals at the $1 \%$ level with in situ observations for the conditions encountered during this field experiment.

The agreement better than $1 \%$ achieved between the measured ATOMMS spectra and a forward microwave propagation model was substantially better than the comparisons with in situ sensors and indicates the very small level of uncertainty associated with the changes in water vapor that ATOMMS measured. Despite our varying both the combinations of signal frequencies used in the retrievals and the reference times, the agreement remained better than $1 \%$, indicating that there is simply very little ambiguity in the retrievals of changes in the path-averaged vapor pressure. This essentially brings laboratory-quality measurements out into the field, a very desirable and sought-after property of any measurement system.

In terms of the number of signal frequencies required to accurately determine the water vapor, we used between 5 and 15 tuned signal frequencies plus a calibration signal at a fixed frequency for the water vapor spectral fits. The agreement and consistency of these results indicate that the amplitudes from just a few tuned frequencies and a fixed frequency amplitude calibration signal are needed to produce water vapor retrievals with very small random and absolute uncertainties. We also note that the spectral sweeps used in the mountaintop experiment were intentionally finely spaced in frequency and therefore slow as well as redundant so that we could assess instrument performance, the absorption and scattering spectra and the performance of the retrievals. Faster spectral sampling, required for LEO-LEO occultations, is readily achievable using a combination of faster switching synthesizers and a smaller number of frequencies to sample the spectrum.

These field measurements of attenuation made near the $183 \mathrm{GHz}$ water vapor absorption line in the presence of rain and liquid clouds enabled us to assess the attenuation due to liquid hydrometeors and the ambiguities associated with them. In terms of raindrop-sized liquid hydrometeors, Mie theory predicts that their attenuation across the $183 \mathrm{GHz}$ band has little dependence on signal frequency. As a result, the attenuation due to rain largely ratioed out when we applied the differential absorption technique to determine the changes in water vapor. According to Mie theory, the attenuation of cloud droplet-sized liquid hydrometeors in the $183 \mathrm{GHz}$ band has a spectral dependence that increases approximately linearly with frequency. However, when we accounted for this anticipated dependence, the fit between the observations and forward calculations from a microwave propagation model became slightly worse. The reasons for this are as yet unclear.

In the eventual LEO configuration, the ATOMMS signals will encounter a wider range of hydrometeors and spectral dependencies across both the high- and low-band frequency bands. For example, the $183 \mathrm{GHz}$ band will profile water vapor at high altitudes through ice clouds that will attenuate the signals via Rayleigh scattering, which depends approximately on the fourth power of the signal frequency. The LEO version of ATOMMS will provide the information necessary to observe and account for such nonvapor effects using at least three simultaneous signal frequencies to place amplitude calibration signals on both the low and high sides of the absorption line and the third frequency on the line. At altitudes at which most liquid hydrometeors are encountered, observations in the $22 \mathrm{GHz}$ band will be used to make water vapor retrievals. The liquid water absorption spectrum across the low-band frequencies is generally more complex than the ice particle scattering across the high-band frequencies. Thus, in order to separate the water vapor absorption from the cloud liquid water absorption, we must observe the amplitudes from at least four low-band frequencies, with at least one of the signal frequencies on the high-frequency side of the $22 \mathrm{GHz}$ absorption line, since the liquid water absorption increases with frequency across the entire low frequency band, while the water vapor absorption is greatest at the line center and will have the opposite frequency dependence on the high-frequency side of the line. Under clear-sky conditions, measurements of three to four simultaneously frequencies will allow evaluation and possibly refinement of the spectroscopy of the 22 and $183 \mathrm{GHz}$ water lines. At least one additional frequency would be required to evaluate and improve spectroscopy when clouds are present.

The ability of ATOMMS signals to penetrate though optical depths up to 17 demonstrated here (which would have reached 19 with more stable synthesizers) and to retrieve water vapor to $1 \%$ under a wide range of atmospheric conditions, ranging from clear to cloudy to rain, is well beyond the capability of radiometric systems, with penetration that is typically limited to optical depths around unity. This large dynamic range allows ATOMMS to retrieve water vapor from the mesosphere into the lower troposphere as its concentration varies by many orders of magnitude. It is also necessary to be able to retrieve water vapor when there is increased attenuation from clouds. The stronger $183 \mathrm{GHz}$ line is used at higher altitudes and the weaker $22 \mathrm{GHz}$ line is used at lower altitudes. A design goal for ATOMMS is to have sufficient dynamic range to achieve a large vertical overlap of the high- and low-band measurements and retrieved profiles. A vertical overlap will provide a valuable crosscheck, since the errors in the low-band and high-band systems will be largely independent. The two bands will have different dependencies and sensitivities to turbulence and spectroscopic uncertainty. In the vertical overlap region the observable high-band frequencies will be far from the line center, while the information from the low-band signals will be from frequencies closer to the line center.

A fundamental goal for weather and climate monitoring, prediction and understanding is all-weather unbiased global sampling. IR systems have substantial biases in their coverage due to the limited ability of IR photons to penetrate through clouds (e.g., Hearty et al., 2014) and its 
$\sim 2 \mathrm{~km}$ vertical resolution is poor in comparison to the verticals scales at which water varies in the atmosphere. While downward-viewing passive microwave systems penetrate through clouds, their vertical resolution is very coarse and their retrievals over land are significantly less accurate than over oceans. GPS RO does provide unbiased global coverage, but is limited by an inability to separate the wet and dry gas contributions from the index of refraction.

ATOMMS is much closer to an all-weather global remote-sensing system that will minimize sampling biases. ATOMMS combines the self-calibration and vertical resolution advantages of occultation systems with relatively easy to interpret observations of signal attenuation through the atmosphere that can be inverted to produce accurate, high vertical resolution profiles of water vapor without a priori constraints. In contrast, passive IR and microwave systems require technically challenging measurements of absolute radiance in orbit, which are fundamentally more difficult to interpret, and retrievals of water vapor are more uncertain, vertically coarse and require a priori constraints. An orbiting ATOMMS system achieves near-absolute, long-term stability for climate monitoring simply by measuring changes in amplitude over the $100 \mathrm{~s}$ duration of LEO-LEO occultations.

Given this present situation, the precise, all-weather retrieval capability of ATOMMS, as demonstrated here, would achieve a major advance in remote sensing of the atmosphere. These results support the prediction that an ATOMMS system in LEO would be a major advance toward achieving the fundamental satellite observing system goals of very high vertical resolution, all-weather temperature and water vapor sounding with very small random and absolute uncertainties, across the entire globe in support of weather prediction, climate monitoring and the quantitative constraints on process needed to improve models. A mission design concept using a constellation of very small ATOMMS satellites using cubesat technology is given in Kursinski et al. (2016b). ATOMMS has the potential to provide global observations from space that approach, and in some ways exceed, the performance of sondes.

Data availability. The data used in this study are available upon request by contacting the lead author. The request shall indicate the purpose of requesting the data and must be related only to the results and analysis included in the current publication. 


\section{Appendix A: In situ observational network required for validation of ATOMMS retrievals}

We now discuss the question regarding the quality, quantity and spacing of in situ observations that would be required to validate the ATOMMS retrievals of changes in vapor pressure with time, which we believe are accurate to within $1 \%$. Chilled mirror hygrometers can reach accuracies of $1 \%$, at least in the laboratory. However, when we discussed validating ATOMMS instruments to $1 \%$ with a chilled mirror hygrometer expert at NCAR, we were told that no in situ measurements can reliably achieve $1 \%$ accuracy out in the field (Holger Vömel, personal communication). Chilled mirrors are also expensive. We purchased one for USD 9000 and even the less accurate miniature ones used on balloons are more than USD 1000 apiece. Therefore, while a series of chilled mirrors could be placed along the path, their accuracy might not be as good as required to achieve $1 \%$. They would likely be the closest to $1 \%$ that are available.

The next consideration is how to satisfy the constraints imposed by the ATOMMS measurements, which include (1) a raised observational path between the instruments sufficiently high above the ground surface to avoid surface reflections and (2) a sufficiently long path length to produce enough absorption to enable precise and accurate water vapor retrievals. To avoid contamination of the water vapor observations by the ground surface, the in situ sensors must be located well above the surface $(\sim 50 \mathrm{~m})$ and close to the signal path, but not so close that they interfere with the ATOMMS signal transmission.

Given the variability of the water vapor along the path, the next question is how closely must the in situ instruments be spaced along the signal path to achieve a specified level of accuracy. We estimated the water vapor variability over the $5.4 \mathrm{~km}$ observation path by computing the root mean square (rms) differences for the three available in situ sensors during the experiment, namely the two sensors at each end of the observation path and data from a sensor in the town of Summerhaven in the valley below the observation path. The rms of the differences between the three in situ sensors and the ATOMMS measurements was approximately $8 \%$ during the period from 14:00 to 15:30, which preceded the first period of heavy rain.

To determine how many in situ sensors would be required to achieve $1 \%$ agreement, we turn to the results of Otarola et al. (2011), who used aircraft measurements to determine how the ratio of the standard deviation of humidity point measurements divided by the path-averaged humidity varies with the path length over which the point measurements are averaged. The Otarola et al. (2011) findings are shown in Fig. A1. The straight line segments in the figure represent power law type behavior. The power law exponent of the lines of $\operatorname{SD}(q) /$ mean $(q)$ in Fig. 9 that pass near the point of $\mathrm{SD} /$ mean $=8 \%$ for a path of $5 \mathrm{~km}$ is approximately 0.35 . Given this power-law exponent and the requirement to keep uncertainties smaller than $1 \%$, the path length required to achieve $\operatorname{SD}(q) / \operatorname{mean}(q)=1 \%$ is approximately $10 \mathrm{~m}$. This result is shown graphically in Fig. A1 by the dashed line that passes through the ATOMMS conditions of SD $/$ mean $=8 \%$ for a path of $5 \mathrm{~km}$.

Thus, in situ sensors, accurate to $1 \%$ each, would need to be placed every $10 \mathrm{~m}$ along a $5.4 \mathrm{~km}$ path to achieve an in situ-based path average consistent with the ATOMMS measurements to the $1 \%$ level. This would require approximately 400 total in situ instruments, a very large number of laboratory quality sensors. It would be difficult, if not impossible, to locate these sensors close enough to the signal propagation path without interfering with the signal itself. Furthermore, if the water vapor variations during the heavy rainfall were still larger than the $8 \%$ variations preceding the heavy rainfall, then still denser in situ sampling would be required.

This immediately raises the question of whether one could actually develop, deploy, operate, maintain and protect such a large number of instruments along an elevated path during the kind of severe weather that was required to achieve the high opacities that were observed. We considered using one or more unmanned aerial vehicles (UAVs) carrying precise humidity, temperature and pressure sensors, making measurements along the path during the ATOMMS measurements. This solution has the advantages of flexibility and relatively low cost, but it is not clear that any existing UAV humidity instrumentation can meet our performance needs. Furthermore, the biggest problem with an UAV approach is simply that the UAVs may not survive the intense convective activity that produced the high optical depths observed during our experiment.

We also considered deploying a series of tethered balloons along the $5.4 \mathrm{~km}$ path. However, the problem again is that during intense convective activity, with heavy rain, lighting, severe winds and downdrafts, the balloons would have been dangerous, potentially starting fires when struck by lightning, with at least a subset being destroyed, and the likelihood that the measurement accuracy required to validate ATOMMS would have been low. Given that sonde humidity sensors are notorious for getting wet during rain, which yields positively biased humidity during and following rainfall; just the rainfall itself would likely have degraded the balloons' measurement accuracy.

We discussed using instrumented towers with experts at NCAR, with experience deploying in situ sensors for field experiments. Towers appear to offer the approach most likely capable of successful, accurate measurements aloft during such extreme weather conditions. However, issues of safety for both the instruments and personnel and environment remain as the towers would certainly act as lightning rods, with the potential to start fires. Furthermore, purchasing and deploying the hundreds of towers of sufficient height required to achieve confirmation at $1 \%$ would be quite expensive.

Assuming an approximate cost of USD 2500 per chilled mirror hygrometer, 400 such instruments would cost 


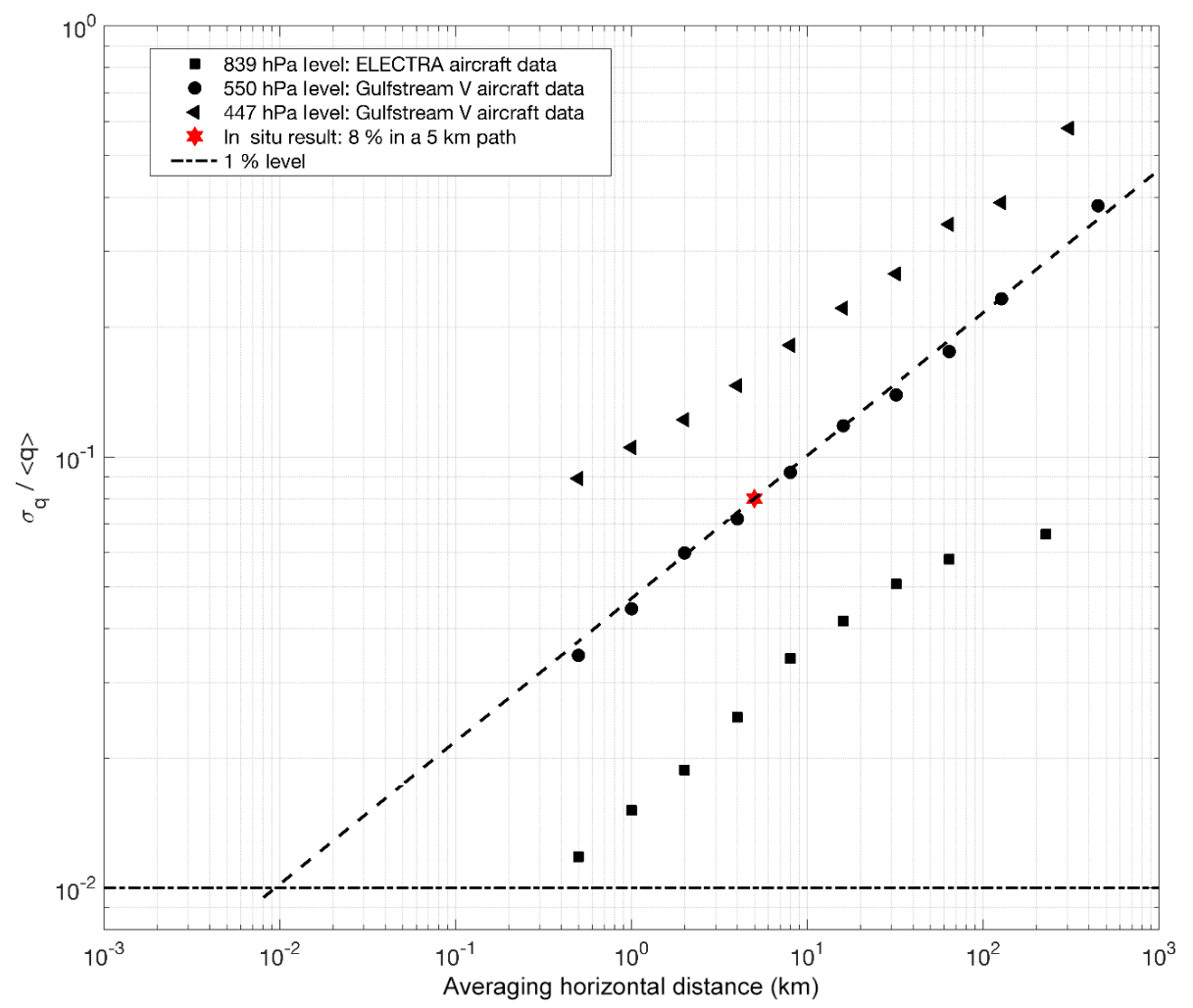

Figure Appendix A1. Ratio of the standard deviation of absolute humidity to the mean absolute humidity based on aircraft data taken at different altitudes, which is indicated by the air pressure along different flight paths. The red star on the dashed line constructed for the $550 \mathrm{hPa}$ altitude observations corresponds with the value calculated from the three in situ sensors operating during the ATOMMS mountaintop experiment (ratio of $8 \%$ for a $5 \mathrm{~km}$ path). The slope of the dashed line corresponds with a power law exponent of 0.35 for the dependence of $\operatorname{SD}(q) /$ mean $(q)$ with the length of the path, which is consistent with Kolomogorov turbulence. Extrapolation of this line to a $\operatorname{SD}(q) / \operatorname{mean}(q)$ value equal to $1 \%$ indicates that in situ observations are required every $10 \mathrm{~m}$ in order to validate the $1 \%$ accuracy of the ATOMMS retrievals. Adapted from Otarola et al. (2011).

USD 1 million. Each would require a data collection system and should be monitored somehow during data collection. The instruments would then need to be placed at the altitude of the ATOMMS signal path, where they would have to be protected from heavy rain, wind and lightning. It is also not clear how many personnel would be required to implement, maintain and operate such an array.

The point of the preceding discussion is that verification by in situ measurements at the level of $1 \%$ uncertainty achieved by the ATOMMS measurements and retrievals out in the field is very difficult (if even possible). As noted, we have not yet identified any practical, cost-effective way to make a sufficient number of in situ observations along the beam path that could have been used to evaluate the ATOMMS retrievals at their level of $1 \%$ precision during periods of intense convection. 
Author contributions. DMW led the interpretation, analysis, and presentation of the results, co-wrote much of the text, participated in field campaigns and instrument testing, and wrote inversion codes for the water vapor retrievals.

ERK was the PI, responsible for the overall ATOMMS investigation and high level instrument design. He led the organization and design of the field campaign, participated in gathering data for this study, participated in interpretation, analysis and discussion of the results and co-wrote much of the text. He also co-developed an approach to use pressure-scale height to determine the path averaged temperatures as discussed in Sect. 4.1 and shown in Fig. 4 and performed the analysis comparing in situ water vapor with the ATOMMS measurements discussed in Sect. 6 and shown in Fig. 8 and analysis of sonde data in Fig. 9.

ACO participated in the field campaign by gathering the data used for the study, helped with data interpretation, analysis, and discussions as well as the preparation of Fig. A1. He suggested and implemented the use of visible cameras along the bore-sight direction of the ATOMMS equipment for monitoring the visible conditions and derivation of optical depths from variations in the intensity of features detected in the visible band images. The acquired data contributed to the generation of Fig. 2. He designed and built two small weather stations based on micro-electro-mechanical (MEM) sensors and Arduino technology for the monitoring of atmospheric conditions next to each of the ATOMMS instruments. The acquired data were used for the analysis shown in Section 4.1, and data shown in Figures 4 and 7. He also designed the HDF5 data structure used to store the ATOMMS raw measurements, programmed the C-language codes available for data conversion from HDF5 to ASCII, C-language/Matlab, codes for computation of the signal amplitudes, and Matlab code for interfacing with the AM model.

MS was key in organizing and making the field campaign gathering of the data for this study a success. He assisted in instrument testing and development and analyzed the optical images to derive the optical depth in the visible bands, which contributed to the generation of Fig. 2.

JMG participated in the field campaign gathering the data for this study and is the RF engineer for the ATOMMS instrument. AY participated in the field campaign gathering the data for this study and is the engineer for the ATOMMS instrument, in particular for its temperature stabilization.

$\mathrm{JH}$ participated in the field campaign gathering the data for this study, developed the instrument enclosures, developed the web cams and software to utilize that data. JH developed the ATOMMS instrument control and data acquisition software. WS developed the digital and control portions of the ATOMMS instrument hardware. HR is the ATOMMS PI at the University of Colorado.

Competing interests. The authors declare that they have no conflict of interest.

Acknowledgements. We want to thank Jeff Kingsley for his support in making critical resources available at the University of Arizona's Steward Observatory needed to complete the ATOMMS instrumentation, and Chris Walker for sharing the Steward Observatory Radio Astronomy Laboratory (SORAL) facilities with us during development of the prototype ATOMMS instrument. We also want to thank Jim Grantham for providing access and modifications to the Mt. Bigelow and Mt. Lemmon facilities to support these observations. We thank David Adams and two anonymous reviewers, whose constructive criticism improved the presentation of this paper considerably. This work was supported by the National Science Foundation Major Research Instrumentation (MRI) Program grant 0723239 and the National Science Foundation, Division of Atmospheric and Geospace Sciences (GEO/AGS) grants 0946411 and 1313563. In particular, we want to thank Jay Fein, program scientist and manager at NSF, who passed away in 2016. Without Jay's insight and relentless effort and support, this research would never have been funded and taken place. A portion of the data analysis was supported by PlanetIQ, Golden, CO.

Edited by: Isaac Moradi

Reviewed by: David Adams and two anonymous referees

\section{References}

Allan, R. P. and Soden, B. J.: Atmospheric warming and the amplification of precipitation extremes, Science, 321, 1481-1484, https://doi.org/10.1126/science.1160787, 2008.

Bohren, C. F. and Huffman, D. R.: Absorption and scattering of light by small particles, New York: Wiley, 82-129, 1983.

Bony, S., Stevens, B., Frierson, D. M. W., Jakob, C., Kageyama, M., and Pincus, R.: Clouds, circulation and climate sensitivity, Nat Geosci., 8, 261-268, https://doi.org/10.1038/ngeo2398, 2015.

Brogniez, H., English, S., Mahfouf, J.-F., Behrendt, A., Berg, W., Boukabara, S., Buehler, S. A., Chambon, P., Gambacorta, A., Geer, A., Ingram, W., Kursinski, E. R., Matricardi, M., Odintsova, T. A., Payne, V. H., Thorne, P. W., Tretyakov, M. Yu., and Wang, J.: A review of sources of systematic errors and uncertainties in observations and simulations at $183 \mathrm{GHz}$, Atmos. Meas. Tech., 9, 2207-2221, https://doi.org/10.5194/amt-9-22072016, 2016.

Calbet, X., Peinado-Galan, N., DeSouza-Machado, S., Kursinski, E. R., Oria, P., Ward, D., Otarola, A., Rípodas, P., and Kivi, R.: Can turbulence within the field of view cause significant biases in radiative transfer modeling at the $183 \mathrm{GHz}$ band?, Atmos. Meas. Tech., 11, 6409-6417, https://doi.org/10.5194/amt11-6409-2018, 2018.

Cardinali, C. and Healy, S.: Impact of GPS radio occultation measurements in the ECMWF system using adjointbased diagnostics, Q. J. Roy. Meteor. Soc., 140: 2315-2320, https://doi.org/10.1002/qj.2300, 2014.

Chen, J., del Genio, A. D., Carlson, B. E., and Bosilovich, M. G.: The Spatiotemporal Structure of Twentieth-Century Climate Variations in Observations and Reanalyses - Part I: Long-Term Trend, J. Climate, 21, 2611-2633, 2008.

Crum, T. D. and Alberty, R. L.: The WSR-88D and the WSR-88D Operational Support Facility, B. Am. Meteorol. Soc., 74, 1669-1688, https://doi.org/10.1175/15200477(1993)074<1669:TWATWO>2.0.CO;2, 1993.

Durran, D. R. and Weyn, J. A.: Thunderstorms Do Not Get Butterflies, B. Am. Meteorol. Soc., 237-244, https://doi.org/10.1175/BAMS-D-15-00070.1, 2016. 
Esau, I. and Sorokina, S.: Climatology of the Arctic Planetary Boundary Layer, in Atmospheric Turbulence, Meteorological Modeling and Aerodynamics, edited by: Lang, P. R. and Lombargo, F. S., 2009, Nova Science Publishers, Inc., ISBN 978-160741-091-1, 2010.

Guan, B. and Waliser, D. E.: Detection of atmospheric rivers: Evaluation and application of an algorithm for global studies, J. Geophys. Res.-Atmos., 120, 12514-12535, https://doi.org/10.1002/2015JD024257, 2015.

Hajj, G. A., Kursinski, E. R., and Walter, S. J.: Examining the Appropriate GPS Science Signal Suitable for Radio Occultation Measurements of Atmospheric Water Vapor, Fall AGU, G22C04, San Francisco, CA, 8-12 December 1997, 1997.

Hardy, K. R., Hajj, G. A., and Kursinski, E. R.: Accuracies of atmospheric profiles obtained from GPS occultations. Int. J. Satell. Commun., 12, 463-473, https://doi.org/10.1002/sat.4600120508, 1994.

Hearty, T. J., Savtchenko, A., Tian, B., Fetzer, E., Yung, Y. L., Theobald, M., Vollmer, B., Fishbein, E., and Won, Y.-I.: Estimating sampling biases and measurement uncertainties of AIRS/AMSU-A temperature and water vapor observations using MERRA reanalysis, J. Geophys. Res.-Atmos., 119, 2725-2741, https://doi.org/10.1002/2013JD021205, 2014.

Held, I. M. and Soden, B. J.: Water vapor feedback and global warming, Annu. Rev. Energ. Env., 25, 441-475, https://doi.org/10.1146/annurev.energy.25.1.441, 2000.

Herman, B., Feng, D., and Xun, X.: GPS Remote Sensing Using a Third Frequency for Water Vapor Profiles of the Atmosphere, Fall AGU, San Francisco, CA, 8-12 December 1997, 1997.

Houze Jr., R. A.: Mesoscale convective systems, Rev. Geophys., 42, RG4003, https://doi.org/10.1029/2004RG000150, 2004.

Itterly, K. F., Taylor, P. C., Dodson, J. B., and Tawfik, A. B.: On the sensitivity of the diurnal cycle in the Amazon to convective intensity, J. Geophys. Res.-Atmos., 121, 8186-8208, https://doi.org/10.1002/2016JD025039, 2016.

Kay, J. E. and Gettelman, A.: Cloud influence on and response to seasonal Arctic sea ice loss, J. Geophys. Res., 114, D18204, https://doi.org/10.1029/2009JD011773, 2009.

Klingebiel, M., de Lozar, A., Molleker, S., Weigel, R., Roth, A., Schmidt, L., Meyer, J., Ehrlich, A., Neuber, R., Wendisch, M., and Borrmann, S.: Arctic low-level boundary layer clouds: in situ measurements and simulations of mono- and bimodal supercooled droplet size distributions at the top layer of liquid phase clouds, Atmos. Chem. Phys., 15, 617-631, https://doi.org/10.5194/acp-15-617-2015, 2015.

Kuo, Y.-H, Zou, X., Chen, S. J., Huang, W., Guo, Y.-R., Anthes, R. A., Exner, M., Hunt, D., Rocken, C., and Sokolovskiy, S.: A GPS/MET Sounding through an Intense Upper-Level Front, B. Am. Meteorol. Soc., 79, 617-626, 1998.

Kursinski, E. R. and Gebhardt, T.: A Method to Deconvolve Errors in GPS RO-Derived Water Vapor Histograms, J. Atmos. Ocean. Technol., 31, 2606-2628, https://doi.org/10.1175/JTECH-D-1300233.1, 2014.

Kursinski, E. R., Hajj, G. A., Schofield, J. T., Linfield, R. P., and Hardy, K. R.: Observing Earth's atmosphere with radio occultation measurements using the Global Positioning System, J. Geophys. Res.-Atmos., 102, 23429-23465, 1997.

Kursinski, E. R., Feng, D., Flittner, D., Hajj, G., Herman, B., Syndergaard, S., Ward, D., and Yunck, T.: A microwave occultation observing system optimized to characterize atmospheric water, temperature and geopotential via absorption, J. Atmos. Ocean. Tech., 19, 1897-1914, 2002.

Kursinski, E. R., Feng, D., Flittner, D., Hajj, G., Herman, B., Romberg, F., Syndergaard, S., Ward, D., and Yunck, T.: An Active Microwave Limb Sounder for Profiling Water Vapor, Ozone, Temperature, Geopotential, Clouds, Isotopes and Stratospheric Winds, in: Occultations for Probing Atmosphere and Climate (OPAC-1), Springer-Verlag, Berlin, ISBN 978-3-540-22350-4, 173-188, 2004.

Kursinski, E. R., Bennett, R. A., Gochis, D., Gutman, S. I., Holub, K. L., Mastaler, R., Minjarez Sosa, C., Minjarez Sosa, I., and van Hove, T.: Water vapor and surface observations in northwestern Mexico during the 2004 NAME enhanced observing period, Geophys. Res. Lett., 35, L03815, https://doi.org/10.1029/2007GL031404, 2008.

Kursinski, E. R., Ward, D., Otarola, A., Frehlich, R., Groppi, C., Albanna, S., Shein, M., Bertiger, W., Pickett, H., and Ross, M.: The active temperature, ozone and moisture microwave spectrometer (ATOMMS), in: New Horizons in Occultation Research, edited by: Steiner, A., Pirscher, B., Foelsche, U., and Kirchengast, G., 295-313, Springer Berlin Heidelberg, 2009.

Kursinski, E. R., Ward, D., Stovern, M., Otarola, A. C., Young, A., Wheelwright, B., Stickney, R., Albanna, S., Duffy, B., Groppi, C., and Hainsworth, J.: Development and testing of the Active Temperature, Ozone and Moisture Microwave Spectrometer (ATOMMS) $\mathrm{cm}$ and $\mathrm{mm}$ wavelength occultation instrument, Atmos. Meas. Tech., 5, 439-456, https://doi.org/10.5194/amt-5439-2012, 2012.

Kursinski, E. R., Ward, D., Otarola, A. C., Kursinski, A. L., and McCormick, C.: Reducing Climate and Weather Prediction Uncertainty via $\mathrm{cm}^{-}$and mm-Wavelength satellite-to-satellite Occultations, White Paper Submitted to 2017 ESAS Decadal Survey In Applications of ATOMMS 072118.docx response to RFI\#1, available at: http: //surveygizmoresponseuploads.s3.amazonaws.com/fileuploads/ 15647/2289356/183-ea6d9954df8cfcbdb60a500c254348c4_ KursinskiEmilR.pdf (last access: 7 March 2019), 2016a.

Kursinski, E. R., Ward, D., Otarola, A. C., McGhee, J., Stovern, M., Sammler, K., Reed, H., Erickson, D., McCormick, C., and Griggs, E.: Atmospheric profiling via satellite to satellite occultations near water and ozone absorption lines for weather and climate. Proc. SPIE 9881, Earth Observing Missions and Sensors: Development, Implementation, and Characterization IV, 98810Z, https://doi.org/10.1117/12.2224038, 2016b.

Leary, C. A.: Temperature and humidity profiles in mesoscale unsaturated downdrafts, J. Atmos. Sci., 37, 1005-1012, 1980.

Liebe, H. J., Hufford, G. A., and Cotton, M. G.: Propagation Modeling of Moist Air and Suspended Water/Ice Particles at Frequencies Below $1000 \mathrm{GHz}$. AGARD Conference Proc. 542, Atmospheric Propagation Effects through Natural and Man-Made Obscurants for Visible to MM-Wave Radiation, 3.1-3.10, 1993.

Liu, C. L., Kirchengast, G., Syndergaard, S., Kursinski, E. R., Sun, Y. O., Bai, W. H., and Du, Q. F.: A review of LEO-LEO occultation techniques using microwave and infrared-laser signals, Adv. Space Res., 60, 2776-2811, https://doi.org/10.1016/j.asr.2017.05.011, 2017.

Otarola, A.C., Querel, R., and Kerber, F.: Precipitable Water Vapor: Considerations on the water vapor scale height, dry bias of 
the radiosonde humidity sensors, and spatial and temporal variability of the humidity field, Contribution in conference "Comprehensive characterization of astronomical sites", 4-10 October 2010, Kislovodsk, Russia, arXiv:1103.3025v1 astro-ph.IM, 1-11, 2011.

Paine, S.: Atmospheric Model am 7.2, available at: http://www.cfa. harvard.edu/ spaine/am/ (last access: 7 March 2019), HarvardSmithsonian Center for Astrophysics, Cambridge, MA, 02138, 2011.

Payne, V. H., Delamere, J. S., Cady-Pereira, K. E., Gamache, R. R., Moncet, J., Mlawer, E. J., and Clough, S. A.: Air broadened half-widths of the 22- and $183-\mathrm{GHz}$ watervapor lines, IEEE T. Geosci. Remote Sens., 46, 3601-3617, https://doi.org/10.1109/TGRS.2008.2002435, 2008.

Rodgers, C. D.: Inverse methods for atmospheric sounding: theory and practice, Vol. 2, World Scientific, 43-61, 2000.

Schiro, K. A., Neelin, J. D., Adams, D. K., and Lintner, B. R.: Deep Convection and Column Water Vapor over Tropical Land versus Tropical Ocean: A Comparison between the Amazon and the Tropical Western Pacific, J. Atmos. Sci., 73, 4043-4063, https://doi.org/10.1175/JAS-D-16-0119.1, 2016.
Sherwood, S. C., Roca, R., Weckwerth, T. M., and Andronova, N. G.: Tropospheric water vapor, convection, and climate, Rev. Geophys., 48, RG2001, https://doi.org/10.1029/2009RG000301, 2010.

Wallace, J. M. and Hobbs, P. V.: Atmospheric Science: An Introductory Survey, Academic, New York, 51-54, 1977.

Wang, J., Dai, A., and Mears, C.: Global Water Vapor Trend from 1988 to 2011 and Its Diurnal Asymmetry Based on GPS, Radiosonde, and Microwave Satellite Measurements, J. Clim., 29, 5205-5222, https://doi.org/10.1175/JCLI-D-15-0485.1, 2016.

Willingham, K. M., Thompson, E. J., Howard, K. W., and Demspey, C. L.: Characteristics of Sonoran Desert Microbursts, Weather Forecast., 26, 94-108, https://doi.org/10.1175/2010WAF2222388.1, 2010.

Wong, S., Fetzer, E. J., Schreier, M., Manipon, G., Fishbein, E. F., Kahn, B. H., Yue, Q., and Irion, F. W.: Cloudinduced uncertainties in AIRS and ECMWF temperature and specific humidity, J. Geophys. Res.-Atmos., 120, 1880-1901, https://doi.org/10.1002/2014JD022440, 2015. 